ACTA AGROBOTANICA

Vol. 59 z. 22006

s. 95108

\title{
The usable taxons in spontaneous flora of railway areas of the central-eastern part of Poland
}

\author{
${ }^{1}$ MAŁGORZATA WRZESIEŃ, ${ }^{2}$ BOŻENA DENISOW \\ ${ }^{1}$ Department of Geobotany, Institute of Biology, Maria Curie Skłodowska University, \\ Akademicka 19., 20033 Lublin, Poland \\ ${ }^{2}$ Department of Botany, University of Agricultural, Akademicka 15, 20950 Lublin, Poland
}

(Received: 10.05.2006)

S u m m a r y

A wide range of ecological conditions on anthropogenic sites makes it easy for different usable species to infiltrate and spread there. The data were collected since 1998. The spontaneous flora of railway areas of the central-eastern part of Poland is composed of already recorded 950 vascular plants, of which 373 are recognized as usable, including 78 dye plants, 162 medicinal, 324 nectariferous or polleniferous taxons. The majority of taxons are distributed in disperse (128 species $34 \%$ ) or occur rarely (96 species 26\%). The common or frequent species constitute $40 \%$ of usable flora of the studied area. Medicinal and melliferous plants occur most frequently on slopes of trenches and railway embankments in nearly natural habitats. They mostly grow as single individuals or form loose and rarely dense patches. The analysis of their geographical status confirms the ultimate prevalence of apophytes over anthropophytes. Considering syntaxonomic structure, there are mainly species typical for phytoceonoses from Molinio-Arrhenatheretea, Artemisietea vulgaris, Stellarietea mediae, Festuco-Brometea, Querco-Fagetea classes.

Key words: railway areas, usable taxons, vascular plants, central eastern part of Poland

\section{INTRODUCTION}

Ever since the beginning of mankind, human being has always taken advantage of medicinal, dye and melliferous plants. Herbaceous species contain different bioactives, e.g. used as a source of pharmacological raw materials which are obtained not only from crops, but still in a big scale from wild taxons. The nectariferous and polleniferous species provide the base for apiaries and create feeding band for different Apoidea. The usable taxons were mainly analysed in natural and semi-natural habitats, rarely in anthropogenicaly transformed areas ( $\mathrm{S} \mathrm{k} \mathrm{r} \mathrm{z} \mathrm{y} \mathrm{c} \mathrm{z} \mathrm{y} \mathrm{ń} \mathrm{s} \mathrm{k} \mathrm{a} \mathrm{and} \mathrm{S}$ t a chowicz, 2003; D r o b i k et al. 2004). 
A wide range of ecological conditions in different anthropogenic habitats makes it easy for a different range of usable species to infiltrate and spread there. The particular richness of the flora is visible on those railway sections which run through open spaces, e.g. fields and meadows where railway lines are situated on embankments, or cross natural hills (Wr z e s i eń and Ś w i ę s, 2006). Such habitats provide conditions both for feeding or convenient nesting and winter surviving of the wild Apoidea. Numerous studies, i.e. based on palynological analysis of bee products, prove that not only crop plants but also the synantropic flora with non-nectarious and anemophilous taxons are significant sources of bee flows (W a r a k o m s k a , 1997; Wróblew s k a, 2002; Denis ow, 2004)

The purpose of the research was to present the share of medicinal, dye and flow plants in spontaneous flora of railways of the central-eastern part of Poland. Also, the value of the object, especially of species recognized as usable, for the protection of natural gene resources was presented. The results can serve as a source of data concerning floristic diversity in the discussed group of plants on the regional scale. It also shows the applicational possibilities of flora originating mainly from railways which have been withdrawn from operation and therefore are of low contamination rate.

\section{MATERIAL AND METHODS}

Data referring to the flora of railway areas were based on field research (published or unpublished) which had been carried out since 1998 on the Lublin Upland, the Roztocze, the Polesie and the Volhynia Upland (Fig. 1). The studied area was divided into 883 incomplete squares of the ATPOL net units which belong to 86 squares of $10 \mathrm{~km}$ long sides ( $\mathrm{Z} \mathrm{a} \mathrm{j} \mathrm{ą} \mathrm{c,} \mathrm{1978).} \mathrm{The} \mathrm{characteristic} \mathrm{concerns} \mathrm{all} \mathrm{types} \mathrm{of}$ habitats related to railway areas, the total length being about $900 \mathrm{~km}$, and includes, inter alia, railway tracks, cargo yards, ridges and embankment slopes, trenches and drainage ditches, and edges of the nearby semi-natural communities, etc.

The usable taxons occurring on the railway areas were selected according to the data available in literature (B r od a, M ow s z ow ic z, 1996; J ę drzejk o et al. 1997; War a k o m s k a, 1997; F a r m a k o pe a VI, 2002; W ró b le w s k a, 2002; Lew k ow ic z-M os iej, 2003). The alphabetical list of species provides taxonomic nomenclature by $\mathrm{M}$ i re $\mathrm{k}$ et al. (2002). The description of each taxon includes its geographic historical status ( $\mathrm{Zaj}$ ą c and $\mathrm{Zaj}$ ą c, 1975; Z a jąc et al. 1998), sinecological group (M t u s z k i e w i c z , 2001), average time of the blooming period (according to own observations), frequency of occurrence in the squares of the ATPOL net units $(10 \times 10 \mathrm{~km})$, location of stations and the degree of density.

\section{RESULTS}

The flora of the studied area is composed of already recorded 950 vascular plants of which 373 are recognized as usable, including 78 dye plants, 162 medicinal, 324 nectariferous or polleniferous (Tab. 1). The taxons under consideration migrate 
Tab. 1

Alphabetical list and characterization of species.

\begin{tabular}{|c|c|c|c|c|c|c|c|}
\hline A & B & $\mathrm{C}$ & D & $\mathbf{E}$ & $\mathbf{F}$ & $\mathbf{G}$ & $\mathbf{H}$ \\
\hline Acernegundo L. & $\mathrm{Ag}$ & - & N.P & $05.04-20.05$ & $\mathrm{~F}$ & $\mathrm{O}, \mathrm{S}$ & 2,3 \\
\hline Acer platanoides L. & Ap & Q-F & B,N,P & $15.04-20.05$ & $\mathrm{D}$ & $0, \mathrm{~S}$ & 1 \\
\hline Acer pseudoplatanus $\mathrm{L}$. & Ap & Q-F & $\mathrm{N}, \mathrm{P}$ & $30.04-30.05$ & $\mathrm{D}$ & $\mathrm{O}, \mathrm{S}$ & 1 \\
\hline Achillea millefolium L. & Ap & M-A & L,B,N,P & $20.05-30.09$ & $\mathrm{C}$ & $\mathrm{O}, \mathrm{S}$ & 2,3 \\
\hline Aegopodium podagraria L. & Ap & Q-F & L,B,N,P & $20.05-10.07$ & $\mathrm{C}$ & $\mathrm{O}$ & 1,2 \\
\hline Aesculus hippocastanum L.. & $\mathrm{Ag}$ & - & L,N,P & $10.05-25.05$ & $\mathrm{R}$ & $\mathrm{O}$ & 1 \\
\hline Aethusa cynapium L. & Arch. & SM & L,N,P & $10.06-20.08$ & $\mathrm{R}$ & $\mathrm{O}$ & 2 \\
\hline Agrimonia eupatoria L. & Ap & F-B & L.B,N,P & $10.06-15.08$ & $\mathrm{C}$ & $\mathrm{O}, \mathrm{S}$ & 1 \\
\hline Ajuga reptans $\mathrm{L}$. & Ap & - & N,P & $05.05-10.07$ & $\mathrm{D}$ & $\mathrm{O}$ & 2 \\
\hline Alchemilla monticola Opiz & Ap & M-A & $\mathrm{L}$ & - & $\mathrm{R}$ & $\mathrm{O}$ & 2 \\
\hline Allium angulosum L. & Ap & M-A & $\mathrm{N}, \mathrm{P}$ & $15.07-15.08$ & $\mathrm{D}$ & $\mathrm{O}, \mathrm{S}$ & 1 \\
\hline Allinm oleraceum L. & Ap & $\mathrm{F}-\mathrm{B}$ & N.P & $05.07-10.08$ & $\mathrm{D}$ & $\mathrm{O}, \mathrm{S}$ & 1 \\
\hline Allium vineale $\mathrm{L}$. & Ap & $\mathrm{F}-\mathrm{B}$ & $\mathrm{N}, \mathrm{P}$ & $15.07-10.08$ & $\mathrm{R}$ & $\mathrm{O}, \mathrm{S}$ & 1 \\
\hline Almus glutinosa (L.) Gaertn. & $\mathrm{Ap}$ & - & $\mathrm{L}, \mathrm{B}$ & - & $\mathrm{F}$ & $\mathrm{O}$ & 1 \\
\hline Althaea officinalis $\mathrm{L}$. & $\mathrm{Ap}$ & M-A & L,B,N,P & $10.06-20.08$ & $\mathrm{R}$ & $\mathrm{O}$ & 1 \\
\hline Anchusa arvensis L. & Ap & $\mathrm{SM}$ & $\mathrm{N}, \mathrm{P}$ & $10.06-20.08$ & $\mathrm{~F}$ & $\mathrm{O}, \mathrm{S}$ & 1 \\
\hline Anchusa officinalis L. & Ap & $\mathrm{AR}$ & $\mathrm{B}, \mathrm{N}, \mathrm{P}$ & $15.05-30.09$ & D & $\mathrm{O}, \mathrm{S}$ & 1 \\
\hline Anemone nemorosa $\mathrm{L}$. & Ap & Q-F & $\mathrm{P}$ & $15.04-10.05$ & D & $\mathrm{O}$ & 2 \\
\hline Angelica sylvestris L. & Ap & M-A & N,P & $15.07-20.08$ & $\mathrm{R}$ & $\mathrm{O}$ & 1 \\
\hline Anthemis arvensis L. & Arch. & SM & B,N.P & $15.06-20.07$ & $\mathrm{D}$ & $\mathrm{T}, \mathrm{O}$ & 1 \\
\hline Anthemis tinctoria L. & $\mathrm{Ap}$ & $\mathrm{F}-\mathrm{B}$ & B,N,P & $15.06-20.07$ & D & $\mathrm{O}$ & 1 \\
\hline Anthriscus sylvestris (L.) Hoffm. & Ap & $\mathrm{AR}$ & B,N,P & $15.05-15.06$ & $\mathrm{~F}$ & $\mathrm{O}, \mathrm{S}$ & 2 \\
\hline Aquilegia vulgaris L. & Ap & - & L.P & $20.05-15.06$ & $\mathrm{R}$ & $\mathrm{O}$ & 1 \\
\hline Arabidopsis thaliana (L.) Heynh. & Ap & $\mathrm{SM}$ & $\mathrm{N}, \mathrm{P}$ & $15.04-15.06$ & $\mathrm{~F}$ & T,O & 1,2 \\
\hline Arabis glabra (L.) Bernh. & Ap & - & N.P & $10.05-20.06$ & $\mathrm{R}$ & $\mathrm{O}$ & 1 \\
\hline Arabis hirsuta (L.) Scop. & Ap & $\mathrm{F}-\mathrm{B}$ & N,P & $10.05-20.06$ & $\mathrm{R}$ & $\mathrm{O}, \mathrm{S}$ & 1 \\
\hline Arctium lappa L. & Ap & $\mathrm{AR}$ & L,B,N,P & $10.07-20.08$ & $\mathrm{~F}$ & $\mathrm{O}, \mathrm{S}$ & 2 \\
\hline Arctium minus (Hill.) Bernh. & Ap & $\mathrm{AR}$ & N.P & $05.07-20.08$ & $\mathrm{D}$ & $\mathrm{O}$ & 1 \\
\hline Anctium tomentosum Mill. & Ap & $\mathrm{AR}$ & L, N,P & $01.07-01.09$ & $\mathrm{~F}$ & $\mathrm{O}, \mathrm{S}$ & 1,2 \\
\hline Armoracia rusticana Gaertn. & Arch. & $\mathrm{AR}$ & $\mathrm{L}$ & - & $\mathrm{D}$ & $\mathrm{O}, \mathrm{S}$ & 1,2 \\
\hline Artemisia absinthium L. & Arch. & $\mathrm{AR}$ & L,B,P & $10.07-30.09$ & $\mathrm{C}$ & $\mathrm{O}, \mathrm{S}$ & $1,2,3$ \\
\hline Artemisia dracunculus L. & Ep & - & $\mathrm{L}$ & - & $\mathrm{R}$ & $\mathrm{S}$ & 2 \\
\hline Artemisia vulgaris L. & $\mathrm{Ap}$ & $\mathrm{AR}$ & L,P & $15.07-20.10$ & $\mathrm{C}$ & $\mathrm{T}, \mathrm{O}, \mathrm{S}$ & $1,2,3$ \\
\hline Asarum europaeum L. & Ap & Q-F & L,P & $10.04-15.05$ & $\mathrm{R}$ & $\mathrm{S}$ & 2 \\
\hline Asparagus officinalis L. & Ap & $\mathrm{F}-\mathrm{B}$ & L.,N,P & $25.05-10.07$ & $\mathrm{R}$ & $\mathrm{S}$ & 1 \\
\hline Aster novi-belgii L. & $\mathrm{Ag}$ & - & N,P & $10.08-20.09$ & D & $\mathrm{O}, \mathrm{S}$ & 2 \\
\hline Astragalus cicer $\mathrm{L}$. & Ap & TG & N.P & $15.05-20.07$ & $\mathrm{R}$ & $\mathrm{O}, \mathrm{S}$ & 2,3 \\
\hline Atriplex patula L. & Ap & $\mathrm{AR}$ & $\mathrm{P}$ & $20.07-20.09$ & $\mathrm{R}$ & $\mathrm{T}, \mathrm{O}$ & 1,2 \\
\hline Ballota nigra L. & Arch. & $\mathrm{AR}$ & L,N,P & $01.07-10.09$ & $\mathrm{C}$ & $\mathrm{T}, \mathrm{O}$ & 1,2 \\
\hline Barbarea vulgaris $\mathrm{R} . \mathrm{Br}$. & Ap & - & $\mathrm{N}, \mathrm{P}$ & $01.05-25.05$ & $\mathrm{D}$ & $\mathrm{T}, \mathrm{O}$ & 1 \\
\hline Bellis perennis $\mathrm{L}$. & Ap & M-A & L,N,P & $20.04-15.07$ & $\mathrm{C}$ & $\mathrm{O}, \mathrm{S}$ & 1 \\
\hline Berberis vulgaris L. & Ap & RP & L,B,N,P, & $10.05-15.06$ & $\mathrm{D}$ & $\mathrm{S}$ & 2 \\
\hline Berteroa incana (L.) DC. & Ap & $\mathrm{AR}$ & N,P & $10.05-30.09$ & $\mathrm{C}$ & $\mathrm{T}, \mathrm{O}, \mathrm{S}$ & $1,2,3$ \\
\hline Betonica officinalis L. & Ap & M-A & L.N,P & $10.06-10.08$ & $\mathrm{D}$ & $\mathrm{S}$ & 1 \\
\hline Betula pendula Roth & Ap & Q-F & L,B & - & $\mathrm{C}$ & $\mathrm{O}, \mathrm{S}$ & 1 \\
\hline Betula pubescens Ehrh & Ap & - & $\mathrm{L}$ & - & $\mathrm{R}$ & $\mathrm{S}$ & 1 \\
\hline Bidens tripartita L. & Ap & BAT & L,B & $20.06-20.08$ & D & $\mathrm{O}$ & 1 \\
\hline Brassica nigra L. & $\mathrm{Ag}$ & - & L,N,P & $10.06-20.08$ & $\mathrm{R}$ & $\mathrm{T}$ & 1 \\
\hline Bryonia alba L. & $\mathrm{Ag}$ & - & $\mathrm{L}$ & - & $\mathrm{R}$ & $\mathrm{O}$ & 2 \\
\hline Bryonia dioica Jacq. & Ep & - & $\mathrm{L}$ & - & $\mathrm{R}$ & $\mathrm{O}$ & 2 \\
\hline
\end{tabular}




\begin{tabular}{|c|c|c|c|c|c|c|c|}
\hline $\mathbf{A}$ & B & C & D & $\mathbf{E}$ & $\mathbf{F}$ & G & $\mathbf{H}$ \\
\hline Bumias orientalis $\mathrm{L}$. & Ep & - & N.P & $05.05-10.06$ & $\mathrm{~F}$ & $\mathrm{~T}, \mathrm{O}, \mathrm{S}$ & 2,3 \\
\hline Calendula arvensis L. & Ep & - & L,N,P & $10.07-15.08$ & $\mathrm{R}$ & $\mathrm{O}$ & 1 \\
\hline Calluna vulgaris (L.) Hull. & Ap & $\mathrm{NC}$ & $\mathrm{L}, \mathrm{B}, \mathrm{N}, \mathrm{P}$ & $05.08-20.09$ & $\mathrm{D}$ & $\mathrm{S}$ & 2 \\
\hline Caltha palustris L. & Ap & M-A & $\mathrm{P}$ & $05.05-25.05$ & $\mathrm{R}$ & $\mathrm{O}$ & 1 \\
\hline Calystegia sepinm (L.) R.Br. & Ap & $\mathrm{AR}$ & N,P & $10.06-10.09$ & $\mathrm{R}$ & $\mathrm{S}$ & 2 \\
\hline Campanula glomerata L. & Ap & $\mathrm{F}-\mathrm{B}$ & N,P & $10.06-01.09$ & $\mathrm{D}$ & $\mathrm{O}$ & 1 \\
\hline Campanula patula L. & Ap & M-A & N,P & $20.05-10.07$ & $\mathrm{~F}$ & $\mathrm{O}$ & 1 \\
\hline Campamula persicifolia $\mathrm{L}$. & Ap & Q-F & $\mathrm{N}, \mathrm{P}$ & $05.07-20.08$ & $\mathrm{D}$ & $\mathrm{O}, \mathrm{S}$ & 1 \\
\hline Campamula rapunculoides L. & Ap & $\mathrm{TG}$ & N,P & $10.06-01.09$ & $\mathrm{~F}$ & $\mathrm{O}$ & 1 \\
\hline Campanula rotundifolia $\mathrm{L}$. & Ap & - & $\mathrm{N}, \mathrm{P}$ & $10.06-01.09$ & $\mathrm{D}$ & $\mathrm{O}$ & 1 \\
\hline Campanula sibirica $\mathrm{L}$. & Ap & F-B & $\mathrm{N}, \mathrm{P}$ & $20.06-15.07$ & $\mathrm{R}$ & $\mathrm{O}, \mathrm{S}$ & 1 \\
\hline Camnabis sativa $\mathrm{L}$. & Ep & - & $\mathrm{L}$ & $01.07-20.08$ & $\mathrm{R}$ & $\mathrm{O}$ & 2 \\
\hline Capsella bursa-pastoris (L.) Med. & Arch. & SM & $\mathrm{L}$ & $10.04-10.10$ & $\mathrm{C}$ & $\mathrm{T}, \mathrm{O}, \mathrm{S}$ & 1 \\
\hline Cardamine pratensis L.s.s. & Ap & M-A & N,P & 10.0510 .06 & $\mathrm{~F}$ & $\mathrm{O}, \mathrm{S}$ & 1 \\
\hline Cardaminopsis arenosa (L.) Hayek & Ap & - & N,P & $20.04-20.05$ & $\mathrm{C}$ & T,O & 1,2 \\
\hline Cardaria draba (L.) Desv. & $\mathrm{Ag}$ & AIR & N.P & $05.05-01.06$ & $\mathrm{~F}$ & $\mathrm{O}, \mathrm{S}$ & 2,3 \\
\hline Carduus acanthoides L. & Arch. & $\mathrm{AR}$ & L,N,P & $20.06-15.09$ & $\mathrm{~F}$ & $\mathrm{O}$ & 1 \\
\hline Cardutus crispus L. & Ap & $\mathrm{AR}$ & N,P & $25.06-01.09$ & $\mathrm{~F}$ & $\mathrm{O}, \mathrm{S}$ & 1,2 \\
\hline Carum carvi L. & Ap & M-A & L,N.P & $10.05-20.06$ & $\mathrm{R}$ & $\mathrm{O}$ & 1 \\
\hline Centaurea cyanus L. & Arch. & SM & $\mathrm{L}, \mathrm{B}, \mathrm{N}, \mathrm{P}$ & $10.06-01.08$ & $\mathrm{D}$ & $\mathrm{O}, \mathrm{S}$ & 1 \\
\hline Centaurea jacea L. & Ap & M-A & N,P & $20.06-20.08$ & $\mathrm{C}$ & $\mathrm{O}$ & 1 \\
\hline Centaurea pannonica (Heuff.) Hayek & Ap & F-B & $\mathrm{N}, \mathrm{P}$ & $20.06-20.08$ & $\mathrm{D}$ & $\mathrm{O}, \mathrm{S}$ & 1 \\
\hline Centaurea scabiosa $\mathrm{L}$. & Ap & F-B & N,P & $20.06-10.09$ & $\mathrm{D}$ & $\mathrm{S}$ & 1,2 \\
\hline Centaurea stoebe $\mathrm{L}$. & Ap & $\mathrm{F}-\mathrm{B}$ & N.P & $25.06-20.08$ & $\mathrm{C}$ & T,O & 1,2 \\
\hline Centaurium pulchellum (Sw) Druce & Ap & IN & $\mathrm{L}$ & - & $\mathrm{R}$ & $\mathrm{O}$ & 1 \\
\hline Cerasus fruticosa Pall & Ap & RP & $\mathrm{B}, \mathrm{N}, \mathrm{P}$ & $20.04-30.04$ & $\mathrm{R}$ & $\mathrm{S}$ & 1,2 \\
\hline $\begin{array}{l}\text { Chamaecytisus ratisbonensis (Schaeff.) } \\
\text { Rothm. }\end{array}$ & Ap & - & N.P & $10.05-15.06$ & D & $\mathrm{S}$ & 2 \\
\hline Chamaenerion angustifolium (L..) Scop. & Ap & EP & L,N,P & $20.06-20.07$ & $\mathrm{~F}$ & $\mathrm{~S}$ & 2 \\
\hline Chamomilla recutita (L.) Rauschert & Arch. & SM & $\mathrm{L}, \mathrm{B}, \mathrm{N}, \mathrm{P}$ & $10.06-20.07$ & $\mathrm{C}$ & T,O & 1,2 \\
\hline Chamomilla suaveolens (Pursh) Rydb. & Ep & M-A & L,N,P & $10.06-20.07$ & $\mathrm{C}$ & $\mathrm{T}, \mathrm{O}$ & 1 \\
\hline Chelidonium majus L. & Ap & AR & L,P & $05.05-10.10$ & $\mathrm{C}$ & T,O & 1 \\
\hline Chenopodium album L. & Ap & $\mathrm{SM}$ & $\mathrm{P}$ & $20.06-20.09$ & $\mathrm{~F}$ & $\mathrm{O}$ & 1,2 \\
\hline Cichorium intybus L. & Arch & $\mathrm{AR}$ & L,N,P & $10.06-01.09$ & $\mathrm{C}$ & $\mathrm{O}, \mathrm{S}$ & 1 \\
\hline Cirsium arvense (L.) Scop. & $\mathrm{Ap}$ & $\mathrm{AR}$ & $\mathrm{B}, \mathrm{N}, \mathrm{P}$ & $30.06-20.08$ & $\mathrm{C}$ & $\mathrm{T}, \mathrm{O}, \mathrm{S}$ & $1,2,3$ \\
\hline Cirsium oleraceum (L.) Scop. & Ap & M-A & $\mathrm{L}, \mathrm{N}, \mathrm{P}$ & $20.06-30.08$ & $\mathrm{D}$ & $\mathrm{S}$ & 1 \\
\hline Cirsium palustre (L.) Scop. & Ap & $\mathrm{M}-\mathrm{A}$ & N,P & $20.06-30.08$ & $\mathrm{R}$ & $\mathrm{S}$ & 1 \\
\hline Cirsium rivulare (Jacq.) All. & Ap & $\mathrm{M}-\mathrm{A}$ & $\mathrm{N}, \mathrm{P}$ & $20.05-30.06$ & $\mathrm{D}$ & $\mathrm{S}$ & 1,2 \\
\hline Cirsium vulgare (Savi.) Ten. & Ap & $\mathrm{AR}$ & N,P & $15.07-15.08$ & $\mathrm{~F}$ & $\mathrm{O}$ & 1 \\
\hline Clematis vitalba $\mathrm{L}$. & $\mathrm{Ag}$ & RP & $\mathrm{P}$ & $20.06-20.08$ & $\mathrm{R}$ & $\mathrm{O}$ & 2 \\
\hline Conium maculatum L. & Arch. & $\mathrm{AR}$ & $\mathrm{L}$ & - & $\mathrm{R}$ & $\mathrm{O}$ & 1,2 \\
\hline Consolida regalis Gray & Arch. & $\mathrm{SM}$ & L.N.P & $10.06-20.07$ & $\mathrm{~F}$ & $\mathrm{~T}, \mathrm{O}$ & 1 \\
\hline Convallaria majalis L. & Ap & - & L,N,P & $05.05-25.05$ & D & $\mathrm{S}$ & 2 \\
\hline Convolvulus arvensis L. & Ap & AIR & L,N,P & $10.06-10.09$ & $\mathrm{C}$ & $\mathrm{T}, \mathrm{O}, \mathrm{S}$ & 2,3 \\
\hline Conyza camadensis (L.) Conquist & Ep & SM & $\mathrm{L}$ & - & $\mathrm{C}$ & $\mathrm{T}, \mathrm{O}$ & $1,2,3$ \\
\hline Coronilla varia L. & Ap & $\mathrm{TG}$ & $\mathrm{B}, \mathrm{N}, \mathrm{P}$ & $10.06-10.08$ & $\mathrm{C}$ & $\mathrm{O}, \mathrm{S}$ & 1,2 \\
\hline Corylus avellana $\mathrm{L}$. & Ap & Q-F & L.P & $20.03-20.04$ & $\mathrm{~F}$ & $\mathrm{O}$ & 1 \\
\hline Crataegus macrocarpa Hegetschw. & Ap & RP & N,P & $15.05-30.07$ & D & $\mathrm{O}, \mathrm{S}$ & 1 \\
\hline Crataegus monogyna Jacq. & Ap & RP & N,P & $15.05-30.05$ & $\mathrm{~F}$ & $\mathrm{O}, \mathrm{S}$ & 1 \\
\hline Datura stramonium L. & Ep & $\mathrm{AR}$ & L.,N,P & $20.06-05.08$ & $\mathrm{R}$ & $\mathrm{O}$ & 1,2 \\
\hline Daucus carota L. & Ap & M-A & L,B,N,P & $20.06-15.09$ & $\mathrm{C}$ & $\mathrm{T}, \mathrm{O}, \mathrm{S}$ & 1,2 \\
\hline
\end{tabular}




\begin{tabular}{|c|c|c|c|c|c|c|c|}
\hline A & B & $\mathbf{C}$ & D & $\mathbf{E}$ & $\mathbf{F}$ & $\mathbf{G}$ & $\mathbf{H}$ \\
\hline Dianthus carthusianorum L. & Ap & $\mathrm{F}-\mathrm{B}$ & N.P & $15.06-20.07$ & D & $\mathrm{S}$ & 1,2 \\
\hline Dianthus superbus L. s.s. & Ap & $\mathrm{M}-\mathrm{A}$ & $\mathrm{N}, \mathrm{P}$ & $20.06-20.07$ & $\mathrm{R}$ & $\mathrm{O}$ & 1,2 \\
\hline Dryopterix filix - mas (L.) Schott H. & Ap & Q-F & $\mathrm{L}$ & - & $\mathrm{D}$ & $\mathrm{O}$ & 1 \\
\hline Echinops sphaerocephalus L. & Ep & $\mathrm{AR}$ & N,P & $10.07-10.08$ & D & $\mathrm{O}$ & 1 \\
\hline Echium vulgare L. & Ap & $\mathrm{AR}$ & $\mathrm{B}, \mathrm{N}, \mathrm{P}$ & $10.06-10.09$ & $\mathrm{C}$ & T,O,S & 1,2 \\
\hline Elymus repens (L.) Gould. & Ap & AIR & $\mathrm{L}$ & - & $\mathrm{C}$ & $\mathrm{T}, \mathrm{O}, \mathrm{S}$ & 2,3 \\
\hline Epilobium hirsutum L. & Ap & $\mathrm{AR}$ & N.P & $10.06-20.09$ & $\mathrm{~F}$ & $\mathrm{~S}$ & 1,2 \\
\hline Epilobium montanum L. & Ap & $\mathrm{AR}$ & N,P & $20.06-30.08$ & $\mathrm{C}$ & $\mathrm{O}, \mathrm{S}$ & 1 \\
\hline Equisetum arvense L. & Ap & AIR & L,B & - & $\mathrm{C}$ & T,O,S & $1,2,3$ \\
\hline Enodium cicutarium (L.) L Her. & Ap & - & $\mathrm{L}, \mathrm{N}, \mathrm{P}$ & $20.05-20.07$ & D & T,O & 1 \\
\hline Euonymus europea L. & Ap & RP & L,B,N,P & $15.05-10.06$ & $\mathrm{~F}$ & $\mathrm{~S}$ & 1 \\
\hline Euonymus verrucosa Scop. & Ap & RP & L,N,P & $15.05-15,06$ & $\mathrm{D}$ & $\mathrm{S}$ & 1 \\
\hline Eupatorium cannabinum L. & Ap & $\mathrm{AR}$ & $\mathrm{L}, \mathrm{B}, \mathrm{N}, \mathrm{P}$ & $20.06-15.08$ & $\mathrm{~F}$ & $\mathrm{~S}$ & 2,3 \\
\hline Euphorbia cyparissias L. & Ap & $\mathrm{F}-\mathrm{B}$ & $\mathrm{L}, \mathrm{B}, \mathrm{N}, \mathrm{P}$ & $01.05-20.05$ & $\mathrm{C}$ & $\mathrm{O}, \mathrm{S}$ & $1,2,3$ \\
\hline Euphorbia esula L. & Ap & - & $\mathrm{N}, \mathrm{P}$ & $20.05-20.07$ & $\mathrm{C}$ & $\mathrm{O}, \mathrm{S}$ & 1,2 \\
\hline Euphorbia helioscopia L. & Arch. & SM & N.P & $10.05-10.06$ & $\mathrm{R}$ & $\mathrm{O}$ & 1 \\
\hline Euphrasia rostkoviana Hayne & Ap & $\mathrm{M}-\mathrm{A}$ & $\mathrm{L}$ & - & D & $\mathrm{S}$ & 1 \\
\hline Fagus sylvatica $\mathrm{L}$. & Ap & Q-F & $\mathrm{L}$ & - & $\mathrm{R}$ & $\mathrm{O}$ & 1 \\
\hline Fallopia convolvulus (L.) A.Love & Arch. & SM & N,P & $20.05-20.06$ & $\mathrm{~F}$ & $\mathrm{O}, \mathrm{S}$ & 2 \\
\hline Ficaria verna Huds. & Ap & Q-F & L,P & $10.04-10.05$ & D & $\mathrm{O}$ & 1,2 \\
\hline Filipendula ulmaria (L.) Maxim. & Ap & M-A & L,B,P & $15.06-20.07$ & $\mathrm{D}$ & $\mathrm{S}$ & 1,2 \\
\hline Filipendula vulgaris Moench & Ap & F-B & $\mathrm{P}$ & $20.06-10.08$ & $\mathrm{D}$ & S & 1,2 \\
\hline Fragaria vesca $\mathrm{L}$. & Ap & EP & L,N,P & $10.05-10.06$ & $\mathrm{C}$ & $\mathrm{O}$ & $1,2,3$ \\
\hline Fragaria viridis L. & Ap & $\mathrm{TG}$ & N.P & $10.05-10.06$ & $\mathrm{D}$ & $\mathrm{O}$ & 1,2 \\
\hline Frangula alnus Mill. & Ap & - & L.B,N,P & $20.05-20.06$ & $\mathrm{D}$ & $\mathrm{O}$ & 1 \\
\hline Fraxinus excelsior L. & Ap & Q-F & $\mathrm{L}$ & - & $\mathrm{F}$ & $\mathrm{O}$ & 1 \\
\hline Fumaria officinalis L. & Arch. & SM & L,B & $20.05-20.08$ & $\mathrm{D}$ & $\mathrm{O}$ & 1,2 \\
\hline Gagea lutea (L.) Ker Gawl. & Ap & Q-F & N.P & $20.04-10.05$ & $\mathrm{R}$ & $\mathrm{O}$ & 1 \\
\hline Galeopsis angustifolia (Ehrh.) Hoffm. & Ap & TR & $\mathrm{N}, \mathrm{P}$ & $15.06-20.08$ & $\mathrm{D}$ & $\mathrm{T}$ & 1,2 \\
\hline Galeopsis pubescens Besser & Ap & $\mathrm{AR}$ & L,N,P & $15.06-01.09$ & $\mathrm{~F}$ & T.O & 1 \\
\hline Galeopsis tetrahit L. & Ap & SM & $\mathrm{L}$ & - & $\mathrm{F}$ & T,O & 1 \\
\hline Galium aparine L. & Ap & $\mathrm{AR}$ & $\mathrm{B}, \mathrm{N}, \mathrm{P}$ & $10.06-15,09$ & $\mathrm{C}$ & $\mathrm{O}, \mathrm{S}$ & 1,2 \\
\hline Galium mollugo L. & Ap & M-A & $\mathrm{B}$ & - & $\mathrm{C}$ & $\mathrm{T}, \mathrm{O}, \mathrm{S}$ & 1,2 \\
\hline Galium odoratum (L.) Scop. & Ap & Q-F & L,B,N,P & $05,05-01,06$ & $\mathrm{D}$ & $\mathrm{O}$ & 1,2 \\
\hline Galium verum $\mathrm{L}$. & Ap & TG & $\mathrm{L}, \mathrm{B}, \mathrm{N}, \mathrm{P}$ & $10.07-20.09$ & $\mathrm{C}$ & $\mathrm{O}, \mathrm{S}$ & $1,2,3$ \\
\hline Genista tinctoria L. & Ap & $\mathrm{NC}$ & $\mathrm{L}, \mathrm{B}, \mathrm{N}, \mathrm{P}$ & $15.06-30.07$ & $\mathrm{D}$ & $\mathrm{O}$ & 2 \\
\hline Geranium palustre L. & Ap & M-A & N.P & $10.06-10.08$ & D & $\mathrm{S}$ & 1,2 \\
\hline Geranium pratense L. & Ap & M-A & N.P & $10.06-10.08$ & $\mathrm{~F}$ & $\mathrm{O}, \mathrm{S}$ & 1,2 \\
\hline Geranium robertiamum L. & Ap & AR & $\mathrm{L}, \mathrm{B}, \mathrm{N}, \mathrm{P}$ & $15.05-20.07$ & $\mathrm{C}$ & T,O & $1,2,3$ \\
\hline Geranium sanguineum & Ap & $\mathrm{TG}$ & N,P & $20.05-10.07$ & $\mathrm{D}$ & $\mathrm{O}, \mathrm{S}$ & 1,2 \\
\hline Geranium sibiricum L. & Ep & - & $\mathrm{N}, \mathrm{P}$ & $20.06-15.08$ & $\mathrm{R}$ & $\mathrm{T}, \mathrm{O}$ & 2 \\
\hline Geramium sylvaticum L. & Ap & $\mathrm{BA}$ & $\mathrm{N}, \mathrm{P}$ & $10.06-20.08$ & $\mathrm{R}$ & $\mathrm{S}$ & 2 \\
\hline Germ urbamum L. & Ap & $\mathrm{AR}$ & L.,B & $01.06-20.06$ & $\mathrm{~F}$ & $0, \mathrm{~S}$ & 1 \\
\hline Glechoma hederacea L. & Ap & $\mathrm{AR}$ & L,N,P & $20.04-10.07$ & $\mathrm{C}$ & $\mathrm{O}$ & 1,2 \\
\hline Hedera helix $\mathrm{L}$. & Ap & - & L,B & - & $\mathrm{R}$ & $\mathrm{O}$ & 2 \\
\hline Helianthus tuberosus L. & $\mathrm{Ag}$ & - & L,N,P & $20.08-15.10$ & $\mathrm{D}$ & $\mathrm{O}$ & 2,3 \\
\hline Helichrysum arenarium (L.) Moench & Ap & $\mathrm{KG}$ & L.B & $20.07-20.08$ & $\mathrm{D}$ & $\mathrm{O}$ & $1,2,3$ \\
\hline Hepatica nobilis Schreb. & Ap & Q-F & L,P & $10.04-30.04$ & $\mathrm{R}$ & $\mathrm{O}$ & 2 \\
\hline $\begin{array}{l}\text { Heracleum mantegazziamum Sommier \& } \\
\text { Levier }\end{array}$ & $\mathrm{Ag}$ & - & $\mathrm{N}, \mathrm{P}$ & $15.06-20.07$ & $\mathrm{R}$ & $\mathrm{O}$ & 3 \\
\hline Heracleum sibiricum L. & Ap & $\mathrm{M}-\mathrm{A}$ & N.P & $10.07-05.08$ & $\mathrm{~F}$ & $\mathrm{O}$ & 1 \\
\hline
\end{tabular}




\begin{tabular}{|c|c|c|c|c|c|c|c|}
\hline$A$ & B & C & D & $\mathbf{E}$ & $\mathbf{F}$ & G & $\mathbf{H}$ \\
\hline Heracleum sphondylium L. & Ap & $\mathrm{M}-\mathrm{A}$ & N,P & $15.06-01.09$ & $\mathrm{~F}$ & $\mathrm{O}$ & 1 \\
\hline Herniaria glabra L. & Ap & $\mathrm{KG}$ & $\mathrm{L}$ & - & $\mathrm{D}$ & $\mathrm{O}$ & 1,2 \\
\hline Humulus lupulus L. & Ap & - & L.P & $10.06-10.07$ & $\mathrm{~F}$ & $\mathrm{O}, \mathrm{S}$ & 2,3 \\
\hline Hyoscyamus niger L. & Arch. & $\mathrm{SM}$ & L,N,P & $01.06-15.07$ & $\mathrm{D}$ & $\mathrm{O}$ & 1 \\
\hline Hypericum perforatum L. & Ap & - & L,B,P & $05.06-30.07$ & $\mathrm{C}$ & $\mathrm{T}, \mathrm{O}, \mathrm{S}$ & 1 \\
\hline Impatiens glandulifera Royle & $\mathrm{Ag}$ & $\mathrm{AR}$ & N.P & $20.07-30.09$ & $\mathrm{R}$ & $\mathrm{S}$ & 2 \\
\hline Impatiens noli-tangere L. & Ap & $\mathrm{Q}-\mathrm{F}$ & $\mathrm{B}, \mathrm{N}, \mathrm{P}$ & $20.07-30.09$ & $\mathrm{D}$ & $\mathrm{O}$ & 1 \\
\hline Impatiens parviflora DC. & $\mathrm{Ag}$ & $\mathrm{AR}$ & $\mathrm{N}, \mathrm{P}$ & $20.07-10.09$ & $\mathrm{~F}$ & $\mathrm{~T}, \mathrm{O}$ & $1,2,3$ \\
\hline Inula helenium L. & $\mathrm{Ag}$ & - & L.N,P & $10.07-20.08$ & $\mathrm{R}$ & $\mathrm{O}$ & 1 \\
\hline Iva xantiifolia Nutt & Ep & $\mathrm{AR}$ & $\mathrm{P}$ & $20.08-20.10$ & $\mathrm{D}$ & $\mathrm{T}, \mathrm{O}$ & 1,2 \\
\hline Jasione montana L. & Ap & $\mathrm{KG}$ & $\mathrm{N}, \mathrm{P}$ & $10.06-30.07$ & $\mathrm{~F}$ & $\mathrm{O}$ & 1,2 \\
\hline Knautia arvensis (L.) J.M.Coult. & Ap & $\mathrm{M}-\mathrm{A}$ & $\mathrm{N}, \mathrm{P}$ & $10.06-30.07$ & $\mathrm{C}$ & $\mathrm{O}, \mathrm{S}$ & 1 \\
\hline Lamium album L. & Arch. & $\mathrm{AR}$ & L,N.P & $20.04-30.09$ & $\mathrm{C}$ & $\mathrm{O}$ & 1 \\
\hline Lamium amplexicaule L. & Arch & $\mathrm{SM}$ & N,P & $01.04-30.06$ & $\mathrm{~F}$ & $\mathrm{O}$ & 1 \\
\hline Lamium purpureum L. & Arch. & SM & N,P & $15.04-01.09$ & $\mathrm{~F}$ & $\mathrm{O}$ & 1 \\
\hline Lathyrus niger (L.) Bernh. & Ap & Q-F & N.P & $15.06-15.07$ & $\mathrm{R}$ & $\mathrm{O}$ & 1,2 \\
\hline Lathyrus palustris L. & $\mathrm{Ap}$ & M-A & $\mathrm{N}, \mathrm{P}$ & $10.07-20.08$ & $\mathrm{R}$ & $\mathrm{S}$ & 2 \\
\hline Lathyrus pratensis L. & Ap & M-A & $\mathrm{B}, \mathrm{N}, \mathrm{P}$ & $15.06-15.07$ & $\mathrm{~F}$ & $\mathrm{O}, \mathrm{S}$ & 2,3 \\
\hline Lathyrus sylvestris L. & Ap & TG & N.P & $05.07-30.07$ & $\mathrm{D}$ & $\mathrm{T}, \mathrm{O}$ & 2,3 \\
\hline Lathyrus tuberosus L. & Arch. & $\mathrm{SM}$ & N.P & $10.07-20.08$ & $\mathrm{~F}$ & $\mathrm{O}, \mathrm{S}$ & 1,2 \\
\hline Lathyrus vermus (L.) Bernh. & Ap & Q-F & N.P & $10.04-20.05$ & $\mathrm{R}$ & $\mathrm{O}$ & 1,2 \\
\hline Leonurus cardiaca L. & Arch. & $\mathrm{AR}$ & L,N,P & $20.06-15.08$ & $\mathrm{~F}$ & $\mathrm{O}, \mathrm{S}$ & 1.2 \\
\hline Lepidium campestre (L.) R.Br. & Arch & - & N,P & $20.05-20.06$ & $\mathrm{D}$ & $\mathrm{T}, \mathrm{O}$ & 1 \\
\hline Ligustrum vulgare L. & $\mathrm{Ag}$ & RP & N.P & $10.05-10.06$ & $\mathrm{R}$ & $\mathrm{O}$ & 2 \\
\hline Lilium martagon L. & Ap & Q-F & $\mathrm{N}, \mathrm{P}$ & $20.05-10.06$ & $\mathrm{R}$ & $\mathrm{S}$ & 1 \\
\hline Limaria vulgaris Mill. & Ap & $\mathrm{AR}$ & L,N,P & $15.06-20.09$ & $\mathrm{C}$ & $\mathrm{T}, \mathrm{O}$ & $1,2,3$ \\
\hline Lithospermum arvense L. & Arch. & $\mathrm{SM}$ & L,N,P & $15.06-20.07$ & $\mathrm{~F}$ & $\mathrm{~T}, \mathrm{O}$ & 1 \\
\hline Lithospermum officinale $\mathrm{L}$. & Ap & - & $\mathrm{L}, \mathrm{B}, \mathrm{N}, \mathrm{P}$ & $15.06-20.07$ & $\mathrm{R}$ & $\mathrm{S}$ & 1 \\
\hline Lonicera tatarica $\mathrm{L}$. & $\mathrm{Ag}$ & - & $\mathrm{N}, \mathrm{P}$ & $20.05-20.06$ & $\mathrm{R}$ & $\mathrm{S}$ & 2 \\
\hline Lotus corniculatus L. & Ap & $\mathrm{M}-\mathrm{A}$ & P.B & $10.05-15.09$ & $\mathrm{C}$ & $\mathrm{O}, \mathrm{S}$ & 1,2 \\
\hline Lychnis flos-cuculi L. & Ap & M-A & N,P & $10.05-25.06$ & $\mathrm{D}$ & $\mathrm{S}$ & 1 \\
\hline Lycium barbarum $L$ & Ep & $\mathrm{AR}$ & L,N,P & $20.05-20.08$ & $\mathrm{R}$ & $\mathrm{S}$ & 2 \\
\hline Lycopodium clavatum $\mathrm{L}$. & Ap & $\mathrm{NC}$ & $\mathrm{L}$ & - & $\mathrm{R}$ & $\mathrm{O}$ & 2 \\
\hline Lysimachia nummularia L. & Ap & $\mathrm{M}-\mathrm{A}$ & L.B,N,P & $15.06-20.07$ & $\mathrm{D}$ & $\mathrm{O}, \mathrm{S}$ & 2 \\
\hline Lythrum salicaria L. & Ap & $\mathrm{M}-\mathrm{A}$ & N.P & $10.07-30.09$ & $\mathrm{D}$ & $\mathrm{S}$ & 1 \\
\hline Malus sylvestris Mill. & Ap & - & $\mathrm{B}, \mathrm{N}, \mathrm{P}$ & $05.05-25.05$ & $\mathrm{R}$ & $\mathrm{O}$ & 1 \\
\hline Malva alcea $\mathrm{L}$. & $\mathrm{Ag}$ & $\mathrm{AR}$ & N,P & $10.07-20.08$ & $\mathrm{D}$ & $\mathrm{O}, \mathrm{S}$ & 1 \\
\hline Malva neglecta Wallr. & Arch. & $\mathrm{SM}$ & N,P & $15.06-01.09$ & $\mathrm{D}$ & $\mathrm{O}$ & 2 \\
\hline Malva sylvestris L. & Arch & $\mathrm{AR}$ & L,N,P & $10.06-20.08$ & $\mathrm{~F}$ & $\mathrm{O}$ & 1 \\
\hline Matricaria maritima L. ssp. imodora & Arch. & $\mathrm{SM}$ & N.P & $10.05-20,09$ & $\mathrm{C}$ & T,O & 1.2 \\
\hline Medicago falcata $\mathrm{L}$. & Ap & $\mathrm{TG}$ & N,P & $10.06-15.09$ & $\mathrm{C}$ & $\mathrm{O}, \mathrm{S}$ & 2,3 \\
\hline Medicago lupulina L. & Ap & - & $\mathrm{N}, \mathrm{P}$ & $10.06-15.08$ & $\mathrm{C}$ & T,O & 1,2 \\
\hline Medicago sativa L. & $\mathrm{Ag}$ & - & N,P & $10.06-15.08$ & $\mathrm{C}$ & $\mathrm{O}$ & 2,3 \\
\hline Medicago $x$ varia Martyn & $\mathrm{Ag}$ & $\mathrm{AR}$ & N.P & $10.06-15.08$ & $\mathrm{~F}$ & $\mathrm{O}$ & 2,3 \\
\hline Melampyrum arvense L. & Ap & $\mathrm{F}-\mathrm{B}$ & N.P & $15.06-25.07$ & $\mathrm{R}$ & $\mathrm{O}$ & 2,3 \\
\hline Melampyrum nemorosum L. & Ap & - & N,P & $15.06-15.08$ & $\mathrm{D}$ & $\mathrm{O}$ & 1,2 \\
\hline Melampyrum pratense L. & Ap & VP & $\mathrm{N}, \mathrm{P}$ & $15.06-15.08$ & $\mathrm{D}$ & $\mathrm{O}$ & 1,2 \\
\hline Melandrium album (Mill.) Garcke & Arch. & $\mathrm{AR}$ & N.P & $20.05-30.09$ & $\mathrm{C}$ & $\mathrm{O}$ & 1 \\
\hline Melilotus alba Medik. & Ap & $\mathrm{AR}$ & N.P & $10.06-15.08$ & $\mathrm{C}$ & $\mathrm{O}, \mathrm{S}$ & 2,3 \\
\hline Melilotus officinalis (L.) Pall. & Ap & $\mathrm{AR}$ & N,P & $01.06-20.07$ & $\mathrm{C}$ & $\mathrm{O}, \mathrm{S}$ & 2,3 \\
\hline Mentha arvensis L. & Ap & - & L,N,P & $20.07-20.09$ & $\mathrm{D}$ & $\mathrm{O}$ & 1 \\
\hline
\end{tabular}




\begin{tabular}{|c|c|c|c|c|c|c|c|}
\hline A & B & C & D & $\mathbf{E}$ & $\mathbf{F}$ & G & $\mathbf{H}$ \\
\hline Mentha longifolia L. & Ap & M-A & N.P & $01.08-20.09$ & $\mathrm{D}$ & $\mathrm{O}$ & 2 \\
\hline Mentha verticillata $\mathrm{L}$. & Ap & - & $\mathrm{N}, \mathrm{P}$ & $20.07-20.09$ & $\mathrm{D}$ & $\mathrm{O}$ & 1 \\
\hline Myosotis arvensis (L.) Hill. & Arch. & SM & $\mathrm{N}, \mathrm{P}$ & $10.05-20.07$ & $\mathrm{C}$ & $\mathrm{T}, \mathrm{O}$ & 1 \\
\hline Myosotis palustris (L.) L.em Rchb. & $\mathrm{Ap}$ & M-A & $\mathrm{N}, \mathrm{P}$ & $10.05-20.07$ & $\mathrm{D}$ & $\mathrm{O}, \mathrm{S}$ & 1,2 \\
\hline Myosotis ramosissima Rochel & Ap & KG & N,P & $15.05-20.06$ & $\mathrm{R}$ & $\mathrm{O}$ & 1 \\
\hline Myosotis sparsiflora Pohl & Ap & - & N,P & $01.06-01.07$ & $\mathrm{R}$ & $\mathrm{S}$ & 2 \\
\hline Myosoton aquaticum (L.) Moench. & Ap & M-A & N,P & $20.05-15.07$ & $\mathrm{D}$ & $\mathrm{S}$ & 1,2 \\
\hline Nepeta calaria L. & Arch. & $\mathrm{AR}$ & L,N,P & $05.07-10.08$ & $\mathrm{R}$ & $\mathrm{O}$ & 1 \\
\hline Nigella arvensis L. & Arch. & - & N,P & $10.07-25.08$ & $\mathrm{R}$ & T,O & 1 \\
\hline Oenothera biennis L.s.s. & Ap & $\mathrm{AR}$ & $\mathrm{L}, \mathrm{N}, \mathrm{P}$ & $15.06-20.08$ & $\mathrm{~F}$ & $\mathrm{O}$ & 1,2 \\
\hline Oenothera casimiri Rostański & Ap & - & $\mathrm{N}, \mathrm{P}$ & $15.06-20.08$ & $\mathrm{D}$ & $\mathrm{O}$ & 1,2 \\
\hline Oenothera parviflora $\mathrm{L}$. & Ep & $\cdot$ & N,P & $15.06-20.08$ & $\mathrm{R}$ & $\mathrm{O}$ & 1,2 \\
\hline Onobrychis arenaria (Kit.) DC & Ap & $\mathrm{F}-\mathrm{B}$ & $\mathrm{N}, \mathrm{P}$ & $15.06-15.07$ & $\mathrm{R}$ & $\mathrm{S}$ & 1 \\
\hline Onobrychis viciifolia Scop. & $\mathrm{Ag}$ & $\mathrm{F}-\mathrm{B}$ & N,P & $15,06-15,07$ & $\mathrm{D}$ & $\mathrm{O}, \mathrm{S}$ & 1 \\
\hline Ononis spinosa $\mathrm{L}$. & Ap & F-B & $\mathrm{L}$ & - & $\mathrm{R}$ & $\mathrm{O}$ & 2 \\
\hline Onopordum acanthium L. & Arch. & $\mathrm{AR}$ & N,P & $15.06-20.07$ & $\mathrm{D}$ & $\mathrm{O}$ & 1 \\
\hline Origanum vulgare L. & Ap & $\mathrm{TG}$ & $\mathrm{L}, \mathrm{B}, \mathrm{N}, \mathrm{P}$ & $01.07-15.08$ & $\mathrm{~F}$ & $\mathrm{O}, \mathrm{S}$ & 1,2 \\
\hline Oxalis acetosella $\mathrm{L}$. & Ap & - & $\mathrm{L}$ & - & $\mathrm{D}$ & $\mathrm{O}$ & 1,2 \\
\hline Padus avium Mill. & Ap & Q-F & L,B,N,P & $20.04-20.05$ & $\mathrm{D}$ & $\mathrm{O}$ & 1 \\
\hline Padis serotina (Ehrh.) Borkh. & $\mathrm{Ag}$ & - & $\mathrm{L}$ & - & $\mathrm{F}$ & $\mathrm{O}$ & 1 \\
\hline Papaver argemone L. & Arch. & SM & $\mathrm{P}$ & $20.05-30.06$ & $\mathrm{D}$ & $\mathrm{O}$ & $1,2,3$ \\
\hline Papaver rhoeas L. & Arch. & SM & $\mathrm{L}, \mathrm{B}, \mathrm{P}$ & $20.05-10.07$ & $\mathrm{~F}$ & $\mathrm{O}$ & $1,2,3$ \\
\hline Pastinaca sativa $\mathrm{L}$. & Arch. & M-A & L.B,N,P & $01.07-10.08$ & $\mathrm{C}$ & $\mathrm{T}, \mathrm{O}, \mathrm{S}$ & 1,2 \\
\hline $\begin{array}{l}\text { Petasites hybridus (L.) P.Gaernt., B.Mey. } \\
\text { \& Scherb. }\end{array}$ & Ap & AR & L,N,P & $15.04-15.05$ & $\mathrm{R}$ & $\mathrm{S}$ & 2 \\
\hline Peucedanum oreoselimum (L.) Moench & Ap & $\mathrm{TG}$ & $\mathrm{L}$ & - & $\mathrm{D}$ & $\mathrm{O}, \mathrm{S}$ & 1,2 \\
\hline Pimpinella saxifraga $\mathrm{L}$. & Ap & - & L,N,P & $15.06-30.09$ & $\mathrm{C}$ & $\mathrm{O}, \mathrm{S}$ & 1,2 \\
\hline Plantago arenaria Waldst. and Kit. & Ap & KG & $\mathrm{L}$ & - & $\mathrm{D}$ & $\mathrm{T}$ & 1,2 \\
\hline Plantago lanceolata L. & Ap & M-A & $\mathrm{L}, \mathrm{P}$ & $15.05-10.09$ & $\mathrm{C}$ & $\mathrm{O}, \mathrm{S}$ & 1 \\
\hline Plantago major $\mathrm{L}$. & Ap & M-A & $\mathrm{L}, \mathrm{P}$ & $20.05-15.08$ & $\mathrm{C}$ & $\mathrm{O}, \mathrm{S}$ & $1,2,3$ \\
\hline Polygonum aviculare L. & Ap & SM & L,N,P,B & $10.05-30.10$ & $\mathrm{C}$ & $\mathrm{O}$ & $1,2,3$ \\
\hline Polygonum bistorta L. & Ap & M-A & $\mathrm{L}, \mathrm{B}, \mathrm{N}, \mathrm{P}$ & $10.05-20.06$ & $\mathrm{D}$ & $\mathrm{S}$ & 1 \\
\hline Polygomum hydropiper L. & Ap & - & L,B,N,P & $10.06-15.07$ & $\mathrm{D}$ & $\mathrm{O}$ & 1 \\
\hline Polygonum persicaria $\mathrm{L}$. & Ap & SM & B & - & $\mathrm{D}$ & $\mathrm{O}$ & 1,2 \\
\hline Populus tremula $\mathrm{L}$. & Ap & EP & $\mathrm{L}$ & - & $\mathrm{F}$ & $\mathrm{O}$ & 1 \\
\hline Potentilla anserina L. & Ap & M-A & L,P & $10.05-20.07$ & $\mathrm{~F}$ & $\mathrm{O}$ & $1,2,3$ \\
\hline Potentilla arenaria Borkh. & Ap & $\mathrm{F}-\mathrm{B}$ & $\mathrm{P}$ & $10.05-15.08$ & $\mathrm{~F}$ & $\mathrm{O}, \mathrm{S}$ & 2,3 \\
\hline Potentilla argentea L.s.s. & Ap & $\mathrm{AR}$ & $\mathrm{P}$ & $10.06-15.07$ & $\mathrm{~F}$ & $\mathrm{O}, \mathrm{S}$ & 1,2 \\
\hline Potentilla collina Wibel s.s. & Ap & KG & $\mathrm{P}$ & $10.06-15.07$ & $\mathrm{D}$ & $\mathrm{O}$ & 1,2 \\
\hline Potentilla erecta (L.) Raeusch & Ap & $\mathrm{NC}$ & $\mathrm{L}, \mathrm{B}, \mathrm{N}$ & $05.05-15.09$ & $\mathrm{D}$ & $\mathrm{O}$ & 1 \\
\hline Potentilla recta $\mathrm{L}$. & Ap & - & $\mathrm{P}$ & $10.06-15.07$ & $\mathrm{D}$ & $\mathrm{O}$ & 1 \\
\hline Potentilla reptans $\mathrm{L}$. & Ap & $\mathrm{M}-\mathrm{A}$ & $\mathrm{P}$ & $05.05-10.09$ & $\mathrm{~F}$ & $\mathrm{~T}, \mathrm{O}$ & 2,3 \\
\hline Potentilla rupestris L. & Ap & - & $\mathrm{P}$ & $15,05-15.07$ & $\mathrm{R}$ & $\mathrm{O}$ & 1 \\
\hline Potentilla supina $\mathrm{L}$. & Ap & IN & $\mathrm{P}$ & $10.06-20.08$ & $\mathrm{R}$ & $\mathrm{O}$ & 1.2 \\
\hline Primula veris $L$ & Ap & F-B & L,B,N,P & $10.04-20.05$ & $\mathrm{R}$ & $\mathrm{O}$ & 1 \\
\hline Prumella vulgaris L. & Ap & M-A & $\mathrm{N}, \mathrm{P}$ & $10.05-20.08$ & $\mathrm{~F}$ & $\mathrm{O}, \mathrm{S}$ & 1 \\
\hline Prumus spinosa $\mathrm{L}$. & Ap & RP & L,N,P & $20.04-05.05$ & $\mathrm{~F}$ & $\mathrm{O}, \mathrm{S}$ & $1,2,3$ \\
\hline Prerydium aquilimum (L.) Kuhn & Ap & - & $\mathrm{B}$ & - & $\mathrm{F}$ & $\mathrm{O}, \mathrm{S}$ & 2,3 \\
\hline Pulmonaria obscura Dumort. & Ap & Q-F & N,P & $20.04-20.05$ & $\mathrm{R}$ & $\mathrm{O}, \mathrm{S}$ & 1,2 \\
\hline Pyrus communis L. & Ap & - & $\mathrm{L}, \mathrm{N}, \mathrm{P}$ & $01.05-20.05$ & $\mathrm{D}$ & $0, \mathrm{~S}$ & 1 \\
\hline Quercus robur $\mathrm{L}$. & Ap & - & L.B & - & $\mathrm{D}$ & $\mathrm{O}$ & 1 \\
\hline
\end{tabular}




\begin{tabular}{|c|c|c|c|c|c|c|c|}
\hline A & B & C & D & $\mathbf{E}$ & $\mathbf{F}$ & G & $\mathbf{H}$ \\
\hline Rammculus acris L. & Ap & $\mathrm{M}-\mathrm{A}$ & $\mathrm{P}$ & $10.05-20.07$ & $\mathrm{~F}$ & $\mathrm{O}, \mathrm{S}$ & 1 \\
\hline Ramunculus bulbosus $\mathrm{L}$. & Ap & $\mathrm{F}-\mathrm{B}$ & $\mathrm{P}$ & $15.05-10.06$ & $\mathrm{D}$ & $\mathrm{S}$ & 1 \\
\hline Ramunculus flammula $\mathrm{L}$. & Ap & $\mathrm{SCH}$ & $\mathrm{P}$ & $15.05-10.08$ & $\mathrm{R}$ & $\mathrm{O}$ & 1 \\
\hline Ramunculus lanuginosus L. & Ap & Q-F & $\mathrm{P}$ & $10.05-10.07$ & $\mathrm{R}$ & $\mathrm{O}$ & 1 \\
\hline Ramunculus polyanthemos $\mathrm{L}$. & Ap & Q-F & $\mathrm{P}$ & $10.06-10.08$ & $\mathrm{R}$ & $\mathrm{O}$ & 1 \\
\hline Raminculus repens L. & Ap & M-A & $\mathrm{P}$ & $10.06-10.08$ & $\mathrm{~F}$ & T,O & 1 \\
\hline Ramenculus sardous Crantz & Ap & M-A & $\mathrm{P}$ & $10.05-20.07$ & D & $\mathrm{O}$ & 1 \\
\hline Raphamıs raphanistrum L. & Arch. & - & $\mathrm{P}$ & $10.06-10.10$ & D & T,O & 1 \\
\hline Reseda lutea L. & Ap & $\mathrm{AR}$ & $\mathrm{B}, \mathrm{N}, \mathrm{P}$ & $20.05-20.07$ & $\mathrm{~F}$ & $\mathrm{O}$ & 1,2 \\
\hline Rhamnus cathartica L. & Ap & RP & $\mathrm{L}, \mathrm{B}, \mathrm{N}, \mathrm{P}$ & $10.05-10.06$ & $\mathrm{D}$ & $\mathrm{O}$ & 1 \\
\hline Ribes nigrum $\mathrm{L}$. & Ap & $\mathrm{AG}$ & L,N,P & $20.04-15.05$ & $\mathrm{D}$ & $\mathrm{O}$ & 1 \\
\hline Ribes uva-crispa L. & Ap & - & N.P & $10.04-01.05$ & $\mathrm{R}$ & $\mathrm{T}, \mathrm{O}$ & 1 \\
\hline Robinia pseudoacacia $\mathrm{L}$. & $\mathrm{Ag}$ & - & $\mathrm{L}, \mathrm{B}, \mathrm{N}, \mathrm{P}$ & $10.06-25.06$ & $\mathrm{D}$ & $\mathrm{O}$ & 1 \\
\hline Rorippa austriaca (Crantz) Besser & Ap & M-A & N.P & $01.06-25.06$ & D & $\mathrm{O}, \mathrm{S}$ & 2,3 \\
\hline Rorippa palustris (L.) Besser & Ap & BAT & N.P & $10.05-20.07$ & $\mathrm{D}$ & $\mathrm{O}$ & 1.2 \\
\hline Rorippa sylvestris (L.) Besser & Ap & $\mathrm{M}-\mathrm{A}$ & N.P & $01.06-01.08$ & $\mathrm{D}$ & $\mathrm{O}$ & 1,2 \\
\hline Rosa canina $\mathrm{L}$. & Ap & $\mathrm{RP}$ & L,P & $15.05-15.06$ & D & $\mathrm{O}, \mathrm{S}$ & 2 \\
\hline Rosa jundzillii Beser & Ap & - & $\mathrm{P}$ & $15.05-15.06$ & $\mathrm{R}$ & $\mathrm{S}$ & 2 \\
\hline Rosa majalis Herrm. & Ap & $\cdot$ & $\mathrm{P}$ & $15.05-15.06$ & $\mathrm{R}$ & $\mathrm{O}$ & 2 \\
\hline Rosa multiflora Thunb. & $\mathrm{Ag}$ & - & $\mathrm{P}$ & $20.05-10.06$ & $\mathrm{D}$ & $\mathrm{O}$ & 2,3 \\
\hline Rosa rugosa Thunb. & $\mathrm{Ag}$ & - & $\mathrm{P}$ & $15.05-25.06$ & $\mathrm{~F}$ & $\mathrm{O}, \mathrm{S}$ & 2,3 \\
\hline Rosa tomentosa SM. & Ap & RP & $\mathrm{P}$ & $10.06-10.07$ & $\mathrm{R}$ & $\mathrm{S}$ & 2 \\
\hline Rubus caesius $\mathrm{L}$. & Ap & $\mathrm{AR}$ & $\mathrm{B}, \mathrm{N}, \mathrm{P}$ & $25.05-10.07$ & $\mathrm{C}$ & $\mathrm{T}, \mathrm{O}, \mathrm{S}$ & 2,3 \\
\hline Rubus idaeus $\mathrm{L}$. & Ap & EP & L,N,P & $20.05-10.07$ & $\mathrm{~F}$ & $\mathrm{O}, \mathrm{S}$ & 2,3 \\
\hline Rubus laciniatus Willd & $\mathrm{Ag}$ & - & $\mathrm{N}, \mathrm{P}$ & $20.05-10.07$ & $\mathrm{R}$ & $\mathrm{T}$ & 2 \\
\hline Rubus plicatus Weihe \& Ness & Ap & RP & $\mathrm{L}, \mathrm{N}, \mathrm{P}$ & $20.05-10.07$ & D & T.O & 2,3 \\
\hline Rudbeckia laciniata $\mathrm{L}$ & $\mathrm{Ag}$ & $\mathrm{AR}$ & $\mathrm{N}, \mathrm{P}$ & $20.07-01.09$ & $\mathrm{R}$ & $\mathrm{O}$ & 1 \\
\hline Rumex acetosa L. & Ap & M-A & $\mathrm{P}$ & $10.05-20.07$ & $\mathrm{C}$ & $\mathrm{O}$ & $1,2,3$ \\
\hline Rumex acetosella $\mathrm{L}$. & Ap & $\mathrm{KG}$ & $\mathrm{B}, \mathrm{P}$ & $01.05-10.07$ & $\mathrm{C}$ & $\mathrm{O}$ & 1,2 \\
\hline Rumex confertus Willd. & $\mathrm{Ag}$ & - & $\mathrm{P}$ & $10.07-20.09$ & $\mathrm{~F}$ & $\mathrm{O}, \mathrm{S}$ & 2,3 \\
\hline Rumex crispus L. & $\mathrm{Ap}$ & - & L,P & $15.06-30.07$ & $\mathrm{~F}$ & $\mathrm{O}$ & 1 \\
\hline Rumex obtusifolius L. & Ap & $\mathrm{AR}$ & L,P & $15.06-15.07$ & D & $\mathrm{O}$ & 1,2 \\
\hline Salix alba L. & Ap & SAL & $\mathrm{B}, \mathrm{N}, \mathrm{P}$ & $10.04-10.05$ & $\mathrm{D}$ & $\mathrm{O}$ & 1,2 \\
\hline Salix cinerea $\mathrm{L}$. & Ap & $\mathrm{AG}$ & N,P & $20.03-20.04$ & $\mathrm{~F}$ & $\mathrm{O}, \mathrm{S}$ & 1,2 \\
\hline Salix pentandra $\mathrm{L}$. & $\mathrm{Ap}$ & $\mathrm{AG}$ & N.P & $20.04-20.05$ & $\mathrm{D}$ & $\mathrm{O}$ & 1,2 \\
\hline Salix purpurea $\mathrm{L}$. & Ap & $\mathrm{SAL}$ & L,N,P & $15.03-20.04$ & $\mathrm{D}$ & $\mathrm{O}$ & 1,2 \\
\hline Salvia nemorosa $\mathrm{L}$. & Ap & $\mathrm{F}-\mathrm{B}$ & N.P & $20.06-05.08$ & $\mathrm{R}$ & $\mathrm{S}$ & 2,3 \\
\hline Salvia pratensis $\mathrm{L}$. & Ap & - & $\mathrm{N}, \mathrm{P}$ & $20.06-05.08$ & $\mathrm{D}$ & $\mathrm{S}$ & 1,2 \\
\hline Salvia verticillata $\mathrm{L}$. & Ap & F-B & $\mathrm{N}, \mathrm{P}$ & $15.06-20.07$ & $\mathrm{D}$ & $\mathrm{S}$ & 1,2 \\
\hline Sambucus nigra L. & Ap & EP & $\mathrm{L}, \mathrm{B}, \mathrm{P}$ & $20.05-20.06$ & $\mathrm{~F}$ & $\mathrm{O}, \mathrm{S}$ & 2,3 \\
\hline Sanguisorba officinalis $\mathrm{L}$. & Ap & M-A & L, N,P & $20.06-20.08$ & $\mathrm{D}$ & $\mathrm{O}$ & 1,2 \\
\hline Saponaria officinalis L. & Ap & $\mathrm{AR}$ & L,N.P & $10.06-20.07$ & $\mathrm{~F}$ & $\mathrm{O}, \mathrm{S}$ & 1,2 \\
\hline Sarothammes scoparius L. & Ap & $\mathrm{RP}$ & L,B,N,P & $15.05-15.06$ & $\mathrm{D}$ & $\mathrm{O}, \mathrm{S}$ & 2,3 \\
\hline Scabiosa ochroletica L. & Ap & F-B & N.P & $01.06-17.07$ & $\mathrm{D}$ & $\mathrm{S}$ & 1 \\
\hline Scleranthus anmuis L. & Arch. & SM & $\mathrm{B}$ & - & $\mathrm{D}$ & $\mathrm{O}$ & 1,2 \\
\hline Sedum acre L. & Ap & KG & L,N,P & $15.05-20.07$ & $\mathrm{~F}$ & T,O & $1,2,3$ \\
\hline Sedum maximum (L.) Hoffm. & Ap & - & L,N,P & $10.07-20.09$ & $\mathrm{~F}$ & $\mathrm{O}$ & 2 \\
\hline Sedum reflexum L. & Ap & KG & L.N,P & $15.05-20.07$ & $\mathrm{R}$ & $\mathrm{O}$ & 2 \\
\hline Sedum sexangulare L. & Ap & KG & L,N.P & $15.05-30.07$ & $\mathrm{D}$ & T,O & $1,2,3$ \\
\hline Senecio jacobaea L. & $\mathrm{Ap}$ & - & L, N,P & $01.07-30.08$ & $\mathrm{~F}$ & $\mathrm{O}$ & 1 \\
\hline Senecio vulgaris $\mathrm{L}$. & Arch. & SM & $\mathrm{L}$ & - & $\mathrm{F}$ & T,O & 1,2 \\
\hline
\end{tabular}




\begin{tabular}{|c|c|c|c|c|c|c|c|}
\hline A & B & $\mathbf{C}$ & D & E & $\mathbf{F}$ & G & $\mathbf{H}$ \\
\hline Serratula tinctoria $\mathrm{L}$. & Ap & M-A & $\mathrm{B}$ & - & $\mathrm{R}$ & $\mathrm{O}$ & 1 \\
\hline Sinapis arvensis L. & Arch. & $\mathrm{SM}$ & N.P & $15.05-15.10$ & $\mathrm{~F}$ & $\mathrm{O}$ & 1 \\
\hline Sisymbrium altissimum L. & Ep & SM & $\mathrm{N}, \mathrm{P}$ & $10.06-30.07$ & $\mathrm{D}$ & $\mathrm{T}, \mathrm{O}$ & 1 \\
\hline Sisymbrinm loeselii L. & Ep & $\mathrm{SM}$ & $\mathrm{N}, \mathrm{P}$ & $01.06-20.07$ & $\mathrm{~F}$ & $\mathrm{~T}, \mathrm{O}, \mathrm{S}$ & $1,2,3$ \\
\hline $\begin{array}{l}\text { Sisymbrium wolgense M. Bieb. ex } \\
\text { E. Foum. }\end{array}$ & Ep & - & $\mathrm{N}, \mathrm{P}$ & $01.06-20.07$ & $\mathrm{D}$ & $\mathrm{O}, \mathrm{S}$ & 2,3 \\
\hline Solidago gigantea Aiton & $\mathrm{Ag}$ & $\mathrm{AR}$ & L,B,N,P & $20.07-15.10$ & $\mathrm{C}$ & $0, \mathrm{~S}$ & 2,3 \\
\hline Solidago virgaurea $\mathrm{L}$. & Ap & - & $\mathrm{L}, \mathrm{B}, \mathrm{N}, \mathrm{P}$ & $28.07-10.10$ & $\mathrm{~F}$ & $\mathrm{O}, \mathrm{S}$ & 1,2 \\
\hline Sorbus aucuparia L. & $\mathrm{Ap}$ & - & $\mathrm{L}, \mathrm{B}, \mathrm{N}, \mathrm{P}$ & $10.05-30.05$ & $\mathrm{~F}$ & $\mathrm{O}$ & 1 \\
\hline Stachys anmia L. & Arch. & SM & $\mathrm{N}, \mathrm{P}$ & $10.06-20.09$ & $\mathrm{R}$ & $\mathrm{S}$ & 1 \\
\hline Stachys pahustris L. & Ap & M-A & $\mathrm{N}, \mathrm{P}$ & $10.06-20.09$ & $\mathrm{D}$ & $\mathrm{O}$ & 1 \\
\hline Stachys recta $\mathrm{L}$. & Ap & $\mathrm{F}-\mathrm{B}$ & N.P & $10.06-20.09$ & $\mathrm{R}$ & $\mathrm{S}$ & 1 \\
\hline Stachys sylvatica L. & Ap & Q-F & N.P & $10.06-20.09$ & $\mathrm{R}$ & $\mathrm{O}$ & 1 \\
\hline Symphoricarpos albus (L.) S. F. Blake & $\mathrm{Ag}$ & - & N,P & $10.05-10.06$ & D & $\mathrm{O}$ & 2 \\
\hline Symphytum officinale L. & Ap & - & $\mathrm{L}, \mathrm{B}, \mathrm{N}, \mathrm{P}$ & $15.05-20.08$ & $\mathrm{R}$ & $\mathrm{O}$ & 1 \\
\hline Tanacetum vulgare $\mathrm{L}$. & Ap & $\mathrm{AR}$ & $\mathrm{L}, \mathrm{B}, \mathrm{P}$ & $20.07-01.10$ & $\mathrm{C}$ & $\mathrm{O}, \mathrm{S}$ & $1,2,3$ \\
\hline Taraxacum officinale F.H.Wigg. & Ap & M-A & $\mathrm{L}, \mathrm{B}, \mathrm{N}, \mathrm{P}$ & $05,05-25,05$ & $\mathrm{C}$ & $\mathrm{O}, \mathrm{S}$ & 1 \\
\hline Teucrium chamaedrys L. & Ap & $\mathrm{F}-\mathrm{B}$ & L,N,P & $15.05-20.06$ & $\mathrm{R}$ & $\mathrm{O}$ & 1,2 \\
\hline Thalictrum aquilegizolium L. & Ap & $\mathrm{BA}$ & $\mathrm{P}$ & $01.06-30.06$ & $\mathrm{R}$ & $\mathrm{O}, \mathrm{S}$ & 2 \\
\hline Thalictrum flavum L. & Ap & M-A & L,P & $20.06-20.07$ & $\mathrm{R}$ & $\mathrm{S}$ & 2 \\
\hline Thalictrum hucidum L. & Ap & - & $\mathrm{P}$ & $15.06-15.07$ & $\mathrm{R}$ & $\mathrm{S}$ & 2 \\
\hline Thalictrum mimus $\mathrm{L}$. & Ap & $\mathrm{TG}$ & L.P & $15.06-30.06$ & $\mathrm{D}$ & O.S & 1,2 \\
\hline Thalictrum simplex $\mathrm{L}$. & Ap & $\mathrm{F}-\mathrm{B}$ & $\mathrm{P}$ & $10.06-10.07$ & $\mathrm{R}$ & $\mathrm{S}$ & 2 \\
\hline Thymus marschallianus Willd. & Ap & $\mathrm{F}-\mathrm{B}$ & $\mathrm{N}, \mathrm{P}$ & $10.06-10.07$ & $\mathrm{R}$ & $\mathrm{S}$ & 2 \\
\hline Thymus pulegioides L. & Ap & - & $\mathrm{N}, \mathrm{P}$ & $10.06-10.07$ & $\mathrm{~F}$ & $\mathrm{O}, \mathrm{S}$ & 2 \\
\hline Thymus serpyllum L. & Ap & KG & L,N,P & $10.06-10.07$ & $\mathrm{~F}$ & $\mathrm{O}, \mathrm{S}$ & 2 \\
\hline Tilia cordata Mill. & Ap & Q-F & L.N,P & $05.07-25.07$ & $\mathrm{~F}$ & $\mathrm{O}$ & 1 \\
\hline Tilia platyplyyllos Scop. & Ap & $\mathrm{Q}-\mathrm{F}$ & N.P & $20.06-25.07$ & $\mathrm{D}$ & $\mathrm{O}$ & 1 \\
\hline Trifolium arvense L. & Ap & $\mathrm{KG}$ & N.P & $01.06-30.07$ & $\mathrm{~F}$ & $\mathrm{O}, \mathrm{S}$ & $1,2,3$ \\
\hline Trifolium campestre Schreb. & Ap & $\mathrm{KG}$ & N,P & $01.06-30.07$ & $\mathrm{D}$ & $\mathrm{O}$ & 1,2 \\
\hline Trifolium dubium Sibth. & Ap & M-A & N.P & $01.06-30.07$ & $\mathrm{D}$ & $\mathrm{O}$ & 1 \\
\hline Trifolium fragiferum L. & Ap & M-A & $\mathrm{N}, \mathrm{P}$ & $01.06-30.07$ & $\mathrm{R}$ & $\mathrm{T}, \mathrm{O}$ & 1,2 \\
\hline Trifolium hybridum L. & $\mathrm{Ap}$ & M-A & N.P & $01.06-30.07$ & $\mathrm{D}$ & $\mathrm{O}$ & 1,2 \\
\hline Trifolium medium L. & Ap & $\mathrm{TG}$ & $\mathrm{N}, \mathrm{P}$ & $01.06-30.07$ & $\mathrm{~F}$ & $\mathrm{O}, \mathrm{S}$ & 2,3 \\
\hline Trifolium montanum L. & Ap & M-A & N.P & $01.06-30.07$ & D & $\mathrm{S}$ & 1 \\
\hline Trifolium pratense L. & Ap & M-A & L,N.P & $01.06-30.07$ & $\mathrm{~F}$ & $\mathrm{O}, \mathrm{S}$ & 1,2 \\
\hline Trifolium repens L. & Ap & M-A & B,N,P & $20.05-30.08$ & $\mathrm{C}$ & $\mathrm{T}, \mathrm{O}, \mathrm{S}$ & $1,2,3$ \\
\hline Tussilago farfara L. & $\mathrm{Ap}$ & AIR & L,N,P & $01.04-25.04$ & $\mathrm{~F}$ & $\mathrm{O}, \mathrm{S}$ & 2,3 \\
\hline Urtica dioica $\mathrm{L}$. & Ap & $\mathrm{AR}$ & $\mathrm{B}, \mathrm{L}$ & - & $\mathrm{C}$ & $\mathrm{T}, \mathrm{O}, \mathrm{S}$ & $1,2,3$ \\
\hline laccinium myrtillus L. & Ap & VP & L,N,P & $10.05-30.05$ & $\mathrm{D}$ & $\mathrm{O}$ & 2 \\
\hline laccinium vitis -idaea $\mathrm{L}$. & Ap & VP & L,N.P & $10.05-30.05$ & $\mathrm{D}$ & $\mathrm{O}$ & 2 \\
\hline Valeriana officinalis L. & Ap & $\mathrm{M}-\mathrm{A}$ & $\mathrm{L}$ & - & $\mathrm{D}$ & $\mathrm{O}, \mathrm{S}$ & 1 \\
\hline Ierbascum densiflorum Bertol. & Ap & $\mathrm{AR}$ & $\mathrm{L}, \mathrm{B}, \mathrm{P}$ & $10.06-0.5 .09$ & $\mathrm{D}$ & $\mathrm{O}$ & 1 \\
\hline Verbascum lychnitis L. & Ap & TG & $\mathrm{P}$ & $10.06-05.09$ & $\mathrm{R}$ & $\mathrm{T}, \mathrm{O}$ & 1,2 \\
\hline lerbascum nigrum L. & Ap & EP & $\mathrm{P}$ & $05.07-10.08$ & $\mathrm{D}$ & $\mathrm{O}$ & 1 \\
\hline lerbascum phlomoides L. & Ap & $\mathrm{AR}$ & L.P & $10.06-05.09$ & $\mathrm{~F}$ & $\mathrm{O}$ & 1 \\
\hline lerbascum phoeniceum L. & Ap & $\mathrm{F}-\mathrm{B}$ & $\mathrm{P}$ & $20.05-30.06$ & $\mathrm{D}$ & $\mathrm{O}, \mathrm{S}$ & 1 \\
\hline lérnica officinalis L. & Arch. & $\mathrm{NC}$ & L,N,P & $10.06-30.07$ & $\mathrm{D}$ & $\mathrm{O}$ & 2,3 \\
\hline Iiburmum opulus L. & Ap & RP & L,N,P,B & $10.05-10.06$ & $\mathrm{D}$ & $\mathrm{O}, \mathrm{S}$ & 1,2 \\
\hline Iícia angustifolia L. & $\mathrm{Ap}$ & SM & $\mathrm{N}, \mathrm{P}$ & $10.05-15.08$ & $\mathrm{~F}$ & $\mathrm{~T}, \mathrm{O}$ & 1 \\
\hline licia cassubica L. & Ap & Q-F & N,P & $20.06-20.07$ & $\mathrm{R}$ & $\mathrm{S}$ & 2 \\
\hline
\end{tabular}




\begin{tabular}{|c|c|c|c|c|c|c|c|}
\hline A & B & C & D & $\mathbf{E}$ & $\mathbf{F}$ & G & H \\
\hline licia cracca L. & Ap & M-A & N,P & $10.06-20.08$ & $\mathrm{C}$ & $\mathrm{T}, \mathrm{O}, \mathrm{S}$ & 1,2 \\
\hline licia dumetorum $\mathrm{L}$. & Ap & $\mathrm{TG}$ & N.P & $05.06-20.08$ & $\mathrm{R}$ & $\mathrm{S}$ & 2 \\
\hline licia grandiflora Scop. & $\mathrm{Ag}$ & - & $\mathrm{N}, \mathrm{P}$ & $10.05-20.06$ & $\mathrm{D}$ & $\mathrm{T}, \mathrm{O}$ & 1,2 \\
\hline Iicia hirsuta (L.) S.F.Gray & Arch. & $\mathrm{SM}$ & N,P & $10.05-15.07$ & $\mathrm{~F}$ & $\mathrm{~T}, \mathrm{O}$ & 1,2 \\
\hline licia sativa $\mathrm{L}$. & Arch. & $\mathrm{SM}$ & N,P & $10.06-25.07$ & $\mathrm{D}$ & $\mathrm{O}$ & 1 \\
\hline Vicia sepium L. & Ap & $\mathrm{TG}$ & N,P & $10.05-30.07$ & $\mathrm{D}$ & $\mathrm{O}, \mathrm{S}$ & 1 \\
\hline Iicia sylvatica $\mathrm{L}$. & Ap & TG & N,P & $10.06-15.07$ & $\mathrm{R}$ & $\mathrm{O}$ & 2 \\
\hline licia tenuifolia Roth & Ap & $\mathrm{SM}$ & N,P & $10.06-15.07$ & $\mathrm{R}$ & $\mathrm{O}, \mathrm{S}$ & 2 \\
\hline Iicia tetrasperma (L.) Schreb. & Arch. & $\mathrm{SM}$ & $\mathrm{N}, \mathrm{P}$ & $10.05-15.08$ & $\mathrm{~F}$ & $\mathrm{~T}, \mathrm{O}$ & 1,2 \\
\hline Iicia villosa Roth. & Arch. & $\mathrm{SM}$ & N,P & $10.06-15.07$ & $\mathrm{~F}$ & $\mathrm{O}, \mathrm{S}$ & 1,2 \\
\hline linca minor $\mathrm{L}$. & Ap & - & $\mathrm{L}, \mathrm{N}, \mathrm{P}$ & $15.04-20.05$ & $\mathrm{R}$ & $\mathrm{O}, \mathrm{S}$ & 2 \\
\hline Iincetoxicum hirundinaria Medik. & Ap & $\mathrm{F}-\mathrm{B}$ & $\mathrm{L}$ & - & $\mathrm{R}$ & $\mathrm{S}$ & 2,3 \\
\hline Viola arvensis Murray & Arch. & $\mathrm{SM}$ & N,P & $10.05-30.09$ & $\mathrm{C}$ & $\mathrm{T}, \mathrm{O}$ & $1,2,3$ \\
\hline Viola canina L. & Ap & $\mathrm{NC}$ & N,P & $10.05-10.06$ & $\mathrm{~F}$ & $\mathrm{O}$ & 2 \\
\hline Viola hirta $\mathrm{L}$. & Ap & $\mathrm{TG}$ & $\mathrm{N}, \mathrm{P}$ & $10.04-20.05$ & D & $\mathrm{O}$ & 2 \\
\hline Tiola mirabilis L. & Ap & - & N,P & $10.04-20.05$ & $\mathrm{R}$ & $\mathrm{O}$ & 2 \\
\hline Tiola odorata L. & Ap & $\mathrm{AR}$ & L,N,P & $20.03-15.05$ & $\mathrm{D}$ & $\mathrm{O}$ & 2 \\
\hline Iiola neichenbachiana Jord. ex Boreau & Ap & Q-F & N,P & $05.04-30.05$ & $\mathrm{~F}$ & $\mathrm{O}$ & 1,2 \\
\hline liola riviniana Rchb. & Ap & - & N,P & $10.04-20.05$ & $\mathrm{~F}$ & $\mathrm{O}$ & 1,2 \\
\hline Iiola tricolor L.s.s. & Ap & - & L,N.P & $20.05-15.09$ & $\mathrm{D}$ & $\mathrm{O}$ & 1,2 \\
\hline Viscum album $\mathrm{L}$. & Ap & - & $\mathrm{L}$ & - & $\mathrm{R}$ & $\mathrm{O}$ & 1 \\
\hline
\end{tabular}

Explanations: A species; B historical and geographical groups: Ap apophytes, Arch archaeophytes, Ep epecophytes, Ag agriophytes; C phytosociological unit: AG Alnetea glutinosae, AR Artemisietea vulgaris, AIR Agropyretea intermedio repentis, BA Betulo Adenostyletea, BAT Bidentetea tripartiti, EP Epilobietea angustifolii, F B Festuco Brome tea, IN Isoëto Nanojuncetea, KG Koelerio glaucae Corynephoretea canescentis, M A Molinio Arrhenatheretea, NC Nardo Callunetea, Q F Querco Fagetae, RP Rhamno Prunetea, SAL Salicetea purpureae, SCH Scheuchzerio Carcetea nigrae, SM Stellarietea mediae, TG Trifolio Geranietea sanguinei, VP Vaccinio Piceetea; D usage form: $\mathrm{L}$ medicinal species, $\mathrm{B}$ dye plants, $\mathrm{N}$ nectariferous, $\mathrm{P}$ polleniferous; $\mathbf{E}$ average time of blooming; $\mathrm{F}$ frequency*: $\mathrm{R}$ rare(1 10 stations), $\mathrm{D}$ in disperse (11 30), $\mathbf{F}$ frequent (31 50), C common (more than 51); $\mathbf{G}$ location of stations: $\mathrm{T}$ railway tracks, $\mathrm{O}$ their edges, $\mathrm{S}$ slopes of trenches and railway embankments; $\mathrm{H}$ degree of density: 1 single, 2 loose patches, 3 dense patches. $*$ take into account $10 \mathrm{~km}$ squares.

both along operated railways and those completely or partly withdrawn from operation. The great majority of them are apophytes (284 species $76 \%$ ) typical for phytocenoses from Molinio-Arrhenatheretea, Artemisietea vulgaris, Stellarietea mediae, Festuco-Brometea, Querco-Fagetea classes (Fig. 2). Despite the anthropogenic habitats, alien species were mainly represented by archeophytes (45 species 12\%), and less frequently by agriophytes (28 species $8 \%$ ) and epecophytes (16 species $4 \%$ ). Most of the analysed species occurred on trench and railway embankment slopes, where the soil is slightly modified or natural.

Herbaceous plants used in unconventional treatments or as raw materials by official pharmacology were represented by 162 taxons (43\% of examined species). That proves the share of medicinal taxons both in natural and anthropogenic floristic objects. 


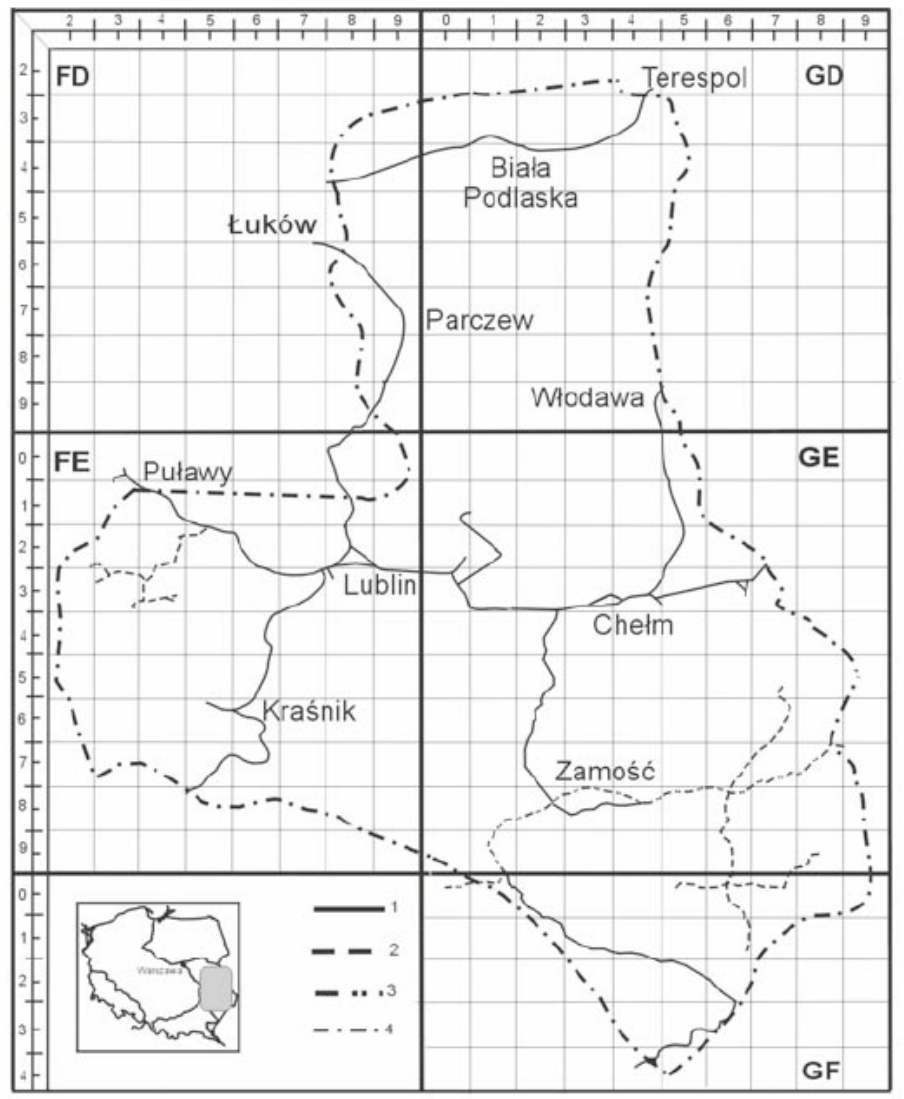

Fig. 1. Study area on the background of ATPOL net squares. 1 - normal, 2 - narrow, 3 - wide gauge railway lines, 4 - border of study area

The most numerous and least frequent, rated as common, were Achillea millefolium, Aegopodium podagraria, Arctium tomentosum, Artemisia absinthium, A. vulgaris, Chamomilla recutita, Chelidonium majus, Daucus carota, Equisetum arvense, Hypericum perforatum, Pimpinella saxifraga, Plantago lanceolata, Polygonum aviculare, Rubus caesius, Rumex acetosa, Taraxacum officinale.

By reason of increasing demand for pharmaceutical raw materials, there are possibilities to obtain the herbaceous taxons from habitats wherever railways are no longer operated. It mainly relates to a narrow gauge railway situated in Lublin and Volhynia Uplands, as well as the railway connecting Lublin with Łuków (Fig.1).

An interesting group was made up of species which provide pollinating entomofauna with nectar and pollen the only source of protein. Especially valuable flow plants found in the studied area were taxons characterised by high density, such as Astragalus cicer, Berteroa incana, Bunias orientalis, Cardaria draba, Cirsium arvense, Linaria vulgaris, Lathyrus sylvestris, Medicago falcata, M. sativa, Prunus spinosa, Rubus caesius, $R$. idaeus. 


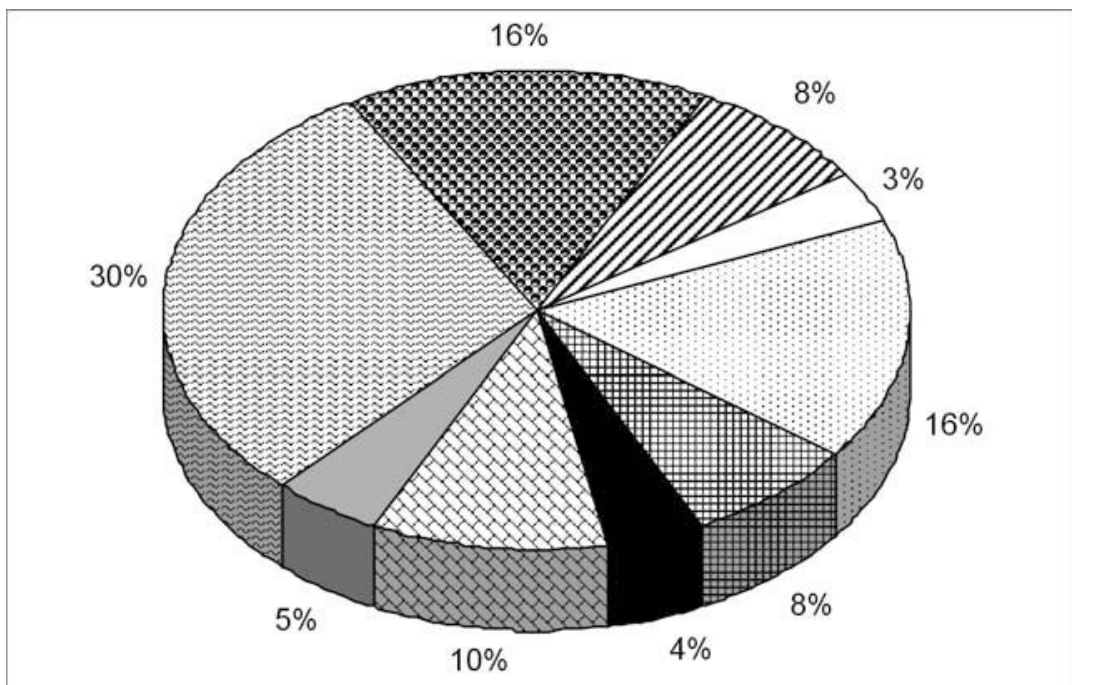

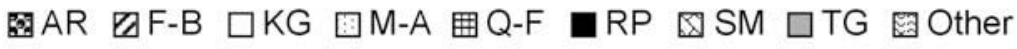

Fig. 2. Number of species in particular syntaxonomical units (explanations like in Tab. 1).

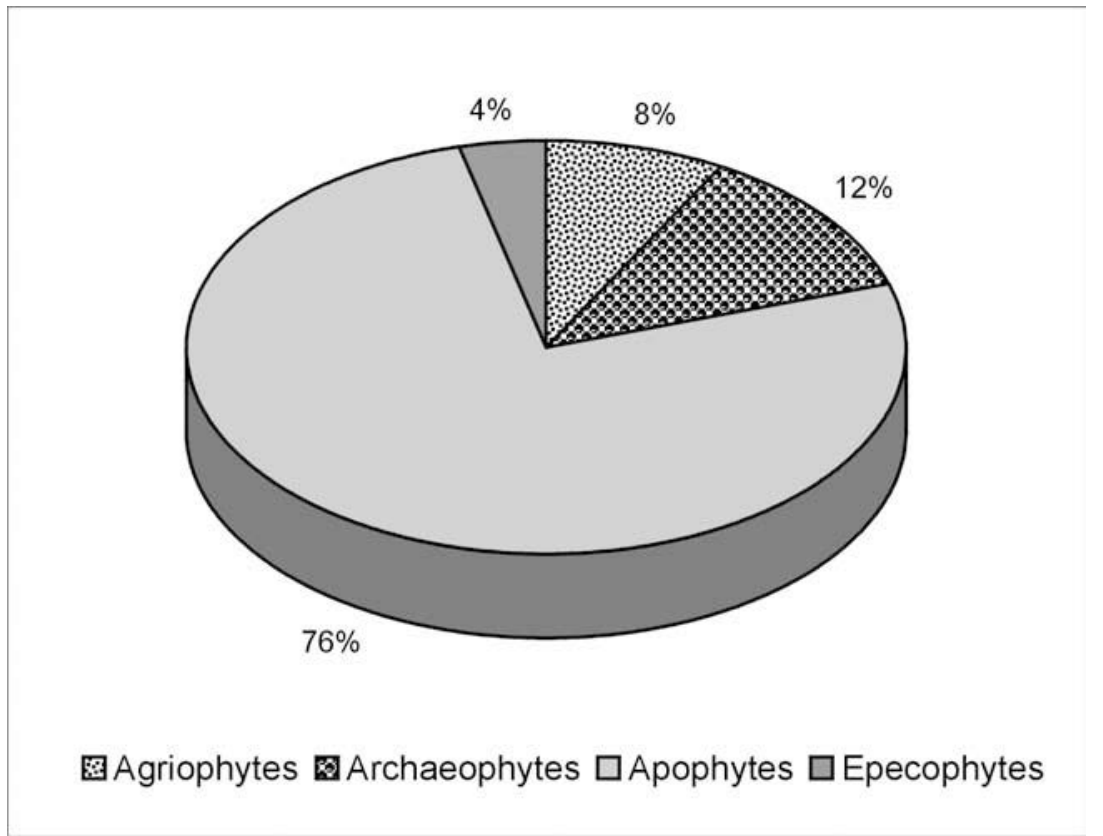

Fig. 3. Share of species in historical and geographical groups. 
In the early spring, when the Apiodea food demand is very high, the Lamium sp., Salix sp., Viola sp. and Anemone nemorosa or Tussilago farfara were in bloom. Among the species which can provide the nectar and pollen in the time of flow gaps, recently common in many parts of Poland, were among others Anchusa officinalis, Astragalus cicer, Berteroa incana, Lychnis flos-cuculi, Lycium barbarum, Polygonum bistorta, Reseda lutea, Sarothamnus scoparius. Such taxons as Helianthus tuberosus, Hieracium umbellatum, and Solidago gigantea, S. virgaurea, blooming from the end of summer till autumn, are very precious, too. The abundance of these flows determines the survival rate of entomofauna during winter and strongly influences their ability for pollination every next spring. The most important feature of beekeeping value of plants is honey and pollen potential. It is worth to obtain species most intensely visited by insects for the detailed study of nectar and pollen abundance and select the most valuable for apiaries.

\section{CONCLUSIONS}

1. The spontaneous flora of railway areas of central-eastern part of Poland comprises 376 usable species ( 78 - dye plants, 162 medicinal, 324 nectariferous or polleniferous).

2. The majority of taxons are distributed in disperse (128 species $34 \%$ ) or occur rarely (96 species 26\%). The common or frequent species constitute $40 \%$ of usable flora of the studied area.

3. Medicinal and melliferous plants occur most frequently on slopes of trenches and railway embankments in nearly natural habitats and create loose or dense patches.

4. Nectariferous and polleniferous taxons provide Apoidea with food from the early spring to the late summer.

5. The floristic diversity of the usable taxons makes it possible to utilize the resources mainly from railway habitats along railways which have been withdrawn from operation. It is highly reasonable to protect natural gene richness of medical and flow plants.

\section{REFERENCES}

De n i s ow B., 2004, Dynamic of blooming and insects visits on several (Brassicaceae $=$ Cruci ferae Juss.) species. J. of Apicultural Sci. 48(2): 1321.

Drobik J., Bacler B., Kowalczyk B., 2004. Rola podstawowych badań florystycznych w ocenie naturalnych zasobów roślin leczniczych. Ann. Acad. Med. Siles. 58(2): 145152.

Farmakopea Polska. Wydanie V I., 2002. Polskie Towarzystwo Farmaceutyczne, War szawa.

Jędrzejko K., Klama H., Żarnowiec J., 1997. Zarys wiedzy o roślinach leczniczych. Śląska Akademia Medyczna, Katowice.

Lewkowicz Mosiej T. 2003., Leksykon roślin leczniczych. Świat Książki, Warszawa.

Matuszkiewicz W. 2001., Przewodnik do oznaczania zbiorowisk roślinnych Polski. Wyd. Nauk. PWN, Warszawa. 
Mirek Z., Piękoś Mirkowa H., Zając A., Zając M., 2002. Flowering plants and pteri dophytes of Poland. A checlist. Biodiversity of Poland 1. W. Szafer Institute of Botany, Polish Academy of Sciences, Kraków.

Skrzyczyńska J., S tachowicz P., 2003. Rośliny zielarskie na odłogach gminy Mielnik. Pam. Puł. 134: 191199.

Warakomska Z., 1997. Obraz pyłkowy wielokwiatowych miodów Lubelszczyzny. LTN, Lublin: 170177.

Wróblews ka A., 2002. Rośliny pożytkowe Podlasia w świetle analizy pyłkowej produktów pszczelich. Rozprawy naukowe Akad. Roln. 264, Lublin.

Wrze si eń M., Ś w ię s F., 2006. Flora i zbiorowiska roślin naczyniowych terenów kolejowych zachodniej części Wyżyny Lubelskiej, Wyd. UMCS, Lublin.

Zaj ą c A., 1978. Założenia metodyczne „Atlasu rozmieszczenia roślin naczyniowych w Polsce”. Wiad. Bot. 22(3): 145155.

Zając A. \& Zając M., (eds.) 2001. Distribution Atlas of Vascular Plants in Poland. Nakładem Pracowni Chorologii Komputerowej Instytutu Botaniki UJ, Kraków.

Zając A., Zając M., Tokarska Guzik B., 1998. Kenophytes in the flora of Poland: list, status and origin. W: Synantropization of plant cover in new Polish research. Phytocoeno sis 10 (N.S.) Suppl. Cartogr. Geobot. 9: 107116.

Zarzycki K., Trzcińska Tacik H., Różański W., Szeląg Z., Wołek J., Korze n i ak U., 2002. Ecological indicator values of vascular plants of Poland. Polish Academy of Sciences, Kraków.

\section{Rośliny użytkowe we florze spontanicznej terenów kolejowych środkowowschodniej Polski}

\section{Streszczenie}

Szeroki wachlarz warunków ekologicznych występujących na siedliskach antropogenicznych umożliwia wnikanie i rozprzestrzenianie się gatunków mających duże znaczenie użytkowe. We florze spontanicznej terenów kolejowych środkowowschodniej Polski odnotowano dotychczas 950 gatunków roślin naczyniowych i wyodrębniono wśród nich 373 gatunki użytkowe (78 rośliny barwierskie, 162 lecznicze, 324 pożytkowe). Większość z nich występuje w rozproszeniu (128 $34 \%$ ) bądź rzadko (96 $26 \%$ ). Taksony pospolite i częste stanowią 40\% analizowanej flory. Rośliny lecznicze i miododajne lokalizują się najczęściej na zboczach wkopów i nasypów kolejowych, gdzie siedliska są słabo przekształcone. Występują pojedynczo lub w luźnym zwarciu, rzadko tworzą zwarte płaty. Analiza ich statusu geograficznego potwierdziła zdecydowaną przewagę gatunków rodzimych (apofitów) nad nowymi przybyszami (antropofitami). Pod względem synekologicznym reprezentują one głównie zbiorowiska z klasy Molinio-Arrhenatheretea, Artemisietea vulgaris, Stellarietea mediae, Festuco-Brometea, Querco-Fagetea. Różnorodność gatunkowa roślin użytkowych daje możliwość wykorzystania ich zasobów na odcinkach wyłączonych z eksploatacji oraz zabezpieczenia żywych zasobów populacyjnych roślin leczniczych i pożytkowych. 